\title{
Switching to non-affine stochastic volatility: A closed-form expansion for the Inverse Gamma model
}

\author{
Nicolas Langrené \\ The Commonwealth Scientific and Industrial Research Organisation \\ Real Options and Financial Risk \\ nicolas.langrene@csiro.au \\ Geoffrey Lee \\ The Commonwealth Scientific and Industrial Research Organisation \\ Real Options and Financial Risk \\ geoffrey.lee@csiro.au \\ Zili Zhu \\ The Commonwealth Scientific and Industrial Research Organisation \\ Real Options and Financial Risk \\ zili.zhu@csiro.au
}

First version: July 8, 2015

This revised version: March 18, 2016

\begin{abstract}
This paper introduces the Inverse Gamma (IGa) stochastic volatility model with time-dependent parameters, defined by the volatility dynamics $d V_{t}=\kappa_{t}\left(\theta_{t}-V_{t}\right) d t+\lambda_{t} V_{t} d B_{t}$.

This non-affine model is much more realistic than classical affine models like the Heston stochastic volatility model, even though both are as parsimonious (only four stochastic parameters). Indeed, it provides more realistic volatility distribution and volatility paths, which translate in practice into more robust calibration and better hedging accuracy, explaining its popularity among practitioners.

In order to price vanilla options with IGa volatility, we propose a closed-form volatility-of-volatility expansion. Specifically, the price of a European put option with IGa volatility is approximated by a Black-Scholes price plus a weighted combination of Black-Scholes greeks, where the weights depend only on the four time-dependent parameters of the model.

This closed-form pricing method allows for very fast pricing and calibration to market data. The overall quality of the approximation is very good, as shown by several calibration tests on real-world market data where expansion prices are compared favorably with Monte Carlo simulation results.

This paper shows that the IGa model is as simple, more realistic, easier to implement and faster to calibrate than classical transform-based affine models. We therefore hope that the present work will foster further research on non-affine models like the Inverse Gamma stochastic volatility model, all the more so as this robust model is of great interest to the industry.
\end{abstract}

Key words: stochastic volatility, Inverse Gamma, volatility expansion, closed-form pricing, lognormal, mean-reverting SABR

JEL Classification: G13, C63, C51, C32, C16, F31, MSC Classification: 91G60, 41A58, 65C20 


\section{Introduction}

The banking industry, especially in equity and foreign exchange, is currently experiencing a shift away from affine stochastic volatility models such as the Heston model, and towards non-affine stochastic volatility models such as the Inverse Gamma model. Non-affine stochastic volatility models have been shown to produce more realistic volatility paths and volatility distributions, to capture more accurately the dynamics of the market implied volatility surfaces, and to produce more reliable calibrations, thus reducing the realized volatility of delta-hedging P\&Ls.

Up until now, the popularity of affine models in spite of their empirical inadequacy has been due to one thing: tractability. Indeed, affine models provide quasi closed-form formulas for vanilla option prices by transform methods, in contrast to non-affine models. The purpose of the present work is to resolve this issue by presenting a fast pricing method for a non-affine stochastic volatility model. More precisely, we develop a closed-form expansion for the price of vanilla options under the non-affine Inverse Gamma stochastic volatility model, defined by the volatility dynamics $d V_{t}=\kappa_{t}\left(\theta_{t}-V_{t}\right) d t+\lambda_{t} V_{t} d B_{t}$.

Implementing this new closed-form expansion is straightforward, and pricing speed is instantaneous. In fact, the closed-form expansion approach is much easier and much faster than the transform methods used for affine models. Moreover, our method is designed to deal naturally with time-dependent parameters. This freedom for the term structure of the model parameters makes the calibration process much easier for various maturities.

We illustrate the accuracy of this closed-form expansion method on several foreign exchange market data sets. The speed and accuracy of the method make it ideal for industry use. The parameters generated through the fast calibration procedure can be used to directly price and hedge options under IGa stochastic volatility, but can also be used in the calibration of more general local-stochastic volatility models.

The paper is organised as follows:

- Section 2 defines the Inverse Gamma stochastic volatility model, discusses similar models in the literature, and discusses the advantages the Inverse Gamma model has over other classical onefactor stochastic volatility models.

- Section 3 provides a closed-form volatility-of-volatility expansion for the price of a European put option under Inverse Gamma stochastic volatility. Furthermore, we provide an algorithm to easily compute the expansion coefficients for piecewise constant parameters, leading to fast calibrations.

- Section 4 provides several numerical tests of the method on foreign exchange market data (AU$\mathrm{D} / \mathrm{USD}, \mathrm{USD} / \mathrm{JPY}, \mathrm{USD} / \mathrm{SGD}$ ). In each example, the Inverse Gamma model is calibrated to the whole implied volatility surface and the expansion prices are compared to Monte Carlo prices. This allows us to assess both calibration error and expansion error.

- Section 5 summarizes the presented methodology and provides some of our plans for future work in this area.

\section{The Inverse Gamma Stochastic volatility model}

This section defines the Inverse Gamma stochastic volatility model and discusses its properties. In this paper we use notations specific to foreign exchange (namely domestic and foreign interest rates), but note that the model itself is not limited to foreign exchange applications and can of course be readily used for other markets (equity, fixed-income, etc.)

\subsection{Definition}

Denote $S_{t}$ and $V_{t}$ as an exchange rate and its instantaneous volatility at time $t$, and $T$ the time horizon considered. The dynamics of the Inverse Gamma (IGa) stochastic volatility model with time-dependent 
parameters is given by

$$
\begin{aligned}
d S_{t} & =\left(r_{d}(t)-r_{f}(t)\right) S_{t} d t+V_{t} S_{t} d W_{t} \\
d V_{t} & =\kappa_{t}\left(\theta_{t}-V_{t}\right) d t+\lambda_{t} V_{t} d B_{t} \\
d\langle W, B\rangle_{t} & =\rho_{t} d t
\end{aligned}
$$

where $\left(W_{t}, B_{t}\right)_{0 \leq t \leq T}$ is a two-dimensional correlated Brownian motion, $r_{d}=\left(r_{d}(t)\right)_{0 \leq t \leq T}$ is the domestic interest rate, and $\bar{r}_{f}=\left(r_{f}(t)\right)_{0 \leq t \leq T}$ is the foreign interest rate. There are four deterministic parameters:

- $\kappa=\left(\kappa_{t}\right)_{0 \leq t \leq T}$ is the rate of mean reversion of the volatility to the level $\theta$.

- $\theta=\left(\theta_{t}\right)_{0 \leq t \leq T}$ is the mean reversion level of the volatility.

- $\lambda=\left(\lambda_{t}\right)_{0 \leq t \leq T}$ is the volatility of volatility.

- $\rho=\left(\rho_{t}\right)_{0 \leq t \leq T}$ is the correlation between the respective Brownian motions of the underlying $S$ and its volatility $V$.

When the parameters are kept constant, the volatility (2.1) is driven by an Inverse Gamma process, yielding an inverse gamma distribution for the stationary distribution of volatility (cf. Appendix A.2). Thus, we denote this model as the Inverse Gamma stochastic volatility model (IGa model in short) with time-dependent parameters ${ }^{1}$.

\subsection{The IGa model in the literature}

A few classes of stochastic volatility models proposed in the literature contain the IGa model with constant parameters as a particular case. To make comparisons simpler, we use the same notations for the parameters of each class $(\kappa, \theta, \lambda, \rho)$ and we remove the drift term from the dynamics of the underlying.

- The Power Arch (or PARCH) stochastic volatility model (Fornari and Mele [2001]),

$$
\begin{aligned}
d S_{t} & =V_{t} S_{t} d W_{t} \\
d V_{t}^{p} & =\kappa\left(\theta-V_{t}^{p}\right) d t+\lambda V_{t}^{p} d B_{t}
\end{aligned}
$$

corresponds to the IGa stochastic volatility model when $p=1$. Remark that $p=2$ corresponds to the GARCH diffusion model (cf. Table 2.1).

- The Double Log-Normal stochastic volatility model (Gatheral [2007, 2008], Henry-Labordère [2009]) with its two cointegrated variance factors:

$$
\begin{aligned}
d S_{t} & =\sqrt{V_{t}} S_{t} d W_{t} \\
d V_{t} & =\kappa\left(V_{t}^{\prime}-V_{t}\right) d t+\lambda V_{t} d B_{t} \\
d V_{t}^{\prime} & =\kappa^{\prime}\left(\theta-V_{t}^{\prime}\right) d t+\lambda^{\prime} V_{t}^{\prime} d B_{t}^{\prime}
\end{aligned}
$$

with correlations between the Brownian motions $W, B$ and $B^{\prime}$. Indeed, the variance formulation of the IGa model can be reformulated as follows:

$$
\begin{aligned}
d S_{t} & =\sqrt{V_{t}} S_{t} d W_{t} \\
d V_{t} & =\left(2 \kappa \theta V_{t}^{\prime}-\left[2 \kappa-\lambda^{2}\right] V_{t}\right) d t+2 \lambda V_{t} d B_{t} \\
d V_{t}^{\prime} & =\kappa\left(\theta-V_{t}^{\prime}\right) d t+\lambda V_{t}^{\prime} d B_{t}
\end{aligned}
$$

which is a particular case of Double Log-Normal stochastic volatility with $100 \%$ correlation between $B$ and $B^{\prime}$.

\footnotetext{
${ }^{1}$ See also Appendix B.
} 
- The $\lambda$-SABR model ${ }^{2}$ (Henry-Labordère [2008] Chapter 6), also known as mean-reverting SABR:

$$
\begin{aligned}
d S_{t} & =V_{t} S_{t}^{\beta} d W_{t} \\
d V_{t} & =\kappa\left(\theta-V_{t}\right) d t+\lambda V_{t} d B_{t}
\end{aligned}
$$

One can see that the IGa model corresponds to the case $\beta=1$, which, along with the case $\beta=1 / 2$, is often considered in practice (see for example Shiraya and Takahashi [2011] or Shiraya and Takahashi [2014]).

- The Generalized Inverse Gamma (GIGa) stochastic volatility model of Ma and Serota [2014]:

$$
\begin{aligned}
d S_{t} & =V_{t} S_{t} d W_{t} \\
d V_{t} & =\kappa\left(\theta V_{t}^{1-\gamma}-V_{t}\right) d t+\lambda V_{t} d B_{t}
\end{aligned}
$$

The special case $\gamma=1$ corresponds to an IGa diffusion for the volatility.

- Finally, the closest model to (2.1) in the literature is the so-called "Log-normal Beta stochastic volatility model" of Sepp [2014, 2015]:

$$
\begin{aligned}
d S_{t} & =V_{t} S_{t} d W_{t} \\
d V_{t} & =\kappa\left(\theta-V_{t}\right) d t+\beta V_{t} d W_{t}+\varepsilon V_{t} d B_{t} \\
d\langle W, B\rangle_{t} & =0
\end{aligned}
$$

The model (2.3) is in fact equivalent to the Inverse Gamma model (2.1), as if $W$ and $B$ are correlated Brownian motions (with correlation $\rho$ ), then $B=\rho W+\sqrt{1-\rho^{2}} W^{\perp}$ where $W^{\perp}$ is another Brownian motion, independent from $W$. Therefore (2.3) is equivalent to (2.1) with $\beta=\lambda \rho$ and $\varepsilon=\lambda \sqrt{1-\rho^{2}}$. For example, the typical equity case $\beta \approx-1$ and $\varepsilon \approx 1$ mentioned in Sepp [2014, $2015]$ corresponds to a volatility of volatility $\lambda=\sqrt{2} \approx 1.41$ and a correlation $\rho=-1 / \sqrt{2} \approx-0.71$.

Other classes of stochastic volatility that contain the IGa model include $d V_{t}=\kappa\left(\theta-V_{t}\right) d t+\lambda V_{t}^{\eta} d B_{t}$ (Jerbi [2011], IGa when $\eta=1$ ) and the general $d V_{t}=\left[q(t) V_{t}^{a}-s(t) V_{t}^{b}\right] d t+l(t) V_{t}^{\gamma+1} d B_{t}$ (Itkin [2013], IGa when $q \equiv \kappa \theta, s \equiv \kappa, l \equiv \lambda, a=0, b=1$, though they focus on the closed forms that can be derived when $a=1$ and $b=2 \gamma+1$, which excludes the IGa model).

This list of models does suggest that the IGa model (2.1) is a sensible and reliable basis to model volatility, but also that the most efficient way to parsimonously enrich the model is not clear yet. This question is left for future research, and the rest of the paper will focus on the IGa model (2.1).

\subsection{Other models}

Over time, many stochastic volatility models have been proposed in the literature. Table 2.1 recalls some classical one factor stochastic volatility models with mean-reversion and correlation between volatility and underlying ${ }^{3}$ with constant parameters. To make comparisons to the IGa model easier, both volatility and variance formulations are given.

\footnotetext{
${ }^{2}$ which, with our notations, is more accurately described as a $\kappa-$ SABR model.

${ }^{3} d\langle W, B\rangle_{t}=\rho d t$ in all models from Table 2.1
} 


\begin{tabular}{|c|c|c|}
\hline Name & $\begin{array}{l}\text { Volatility formulation } \\
d S_{t}=\left(r_{d}-r_{f}\right) S_{t} d t+V_{t} S_{t} d W_{t}\end{array}$ & $\begin{array}{l}\text { Variance formulation } \\
d S_{t}=\left(r_{d}-r_{f}\right) S_{t} d t+\sqrt{V_{t}} S_{t} d W_{t}\end{array}$ \\
\hline Schöbel-Zhu ${ }^{1}$ & $d V_{t}=\kappa\left(\theta-V_{t}\right) d t+\lambda d B_{t}$ & $d V_{t}=\left(\lambda^{2}+2 \kappa \theta \sqrt{V_{t}}-2 \kappa V_{t}\right) d t+2 \lambda \sqrt{V_{t}} d B_{t}$ \\
\hline Heston $^{2}$ & $d V_{t}=\left(\left[\frac{\kappa \theta}{2}-\frac{\lambda^{2}}{8}\right] \frac{1}{V_{t}}-\frac{\kappa}{2} V_{t}\right) d t+\frac{\lambda}{2} d B_{t}$ & $d V_{t}=\kappa\left(\theta-V_{t}\right) d t+\lambda \sqrt{V_{t}} d B_{t}$ \\
\hline $3 / 2$-model ${ }^{3}$ & $d V_{t}=\left(\frac{\kappa \theta}{2} V_{t}-\left[\frac{\kappa}{2}+\frac{\lambda^{2}}{8}\right] V_{t}^{3}\right) d t+\frac{\lambda}{2} V_{t}^{2} d B_{t}$ & $d V_{t}=\kappa\left(\theta V_{t}-V_{t}^{2}\right) d t+\lambda V_{t}^{\frac{3}{2}} d B_{t}$ \\
\hline Log-Normal 4,5 & $d V_{t}=\left(\left[\kappa \theta+\frac{\lambda^{2}}{2}\right] V_{t}-\kappa V_{t} \log \left(V_{t}\right)\right) d t+\lambda V_{t} d B_{t}$ & $d V_{t}=\left(2\left[\kappa \theta+\lambda^{2}\right] V_{t}-\kappa V_{t} \log \left(V_{t}\right)\right) d t+2 \lambda V_{t} d B_{t}$ \\
\hline $\mathrm{GARCH}^{6}$ & $d V_{t}=\left(\frac{\kappa \theta}{2} \frac{1}{V_{t}}-\left[\frac{\kappa}{2}-\frac{\lambda^{2}}{8}\right] V_{t}\right) d t+\frac{\lambda}{2} V_{t} d B_{t}$ & $d V_{t}=\kappa\left(\theta-V_{t}\right) d t+\lambda V_{t} d B_{t}$ \\
\hline $\begin{array}{l}\text { Inverse } \\
\text { Gamma }\end{array}$ & $d V_{t}=\kappa\left(\theta-V_{t}\right) d t+\lambda V_{t} d B_{t}$ & $d V_{t}=\left(2 \kappa \theta \sqrt{V_{t}}-\left[2 \kappa-\lambda^{2}\right] V_{t}\right) d t+2 \lambda V_{t} d B_{t}$ \\
\hline
\end{tabular}

Table 2.1: One factor stochastic volatility models

Among the models listed in Table 2.1, the models of Schöbel-Zhu and Heston are affine, which means that the Fourier transform of the log-price can be computed explicitly. Because of their tractability, affine models have received a lot of attention in the literature, at the expense of the non-affine stochastic volatility models. Unfortunately, empirical analyzes suggest that the dynamics of market volatilities is much better described by non-affine models. Let us illustrate this point by comparing the Heston model and the Inverse Gamma model.

Figure 2.1 displays the stationary distribution of the volatility under the Heston and Inverse Gamma models, with same mean (0.30) and same standard deviation (0.08 on Figures 2.1a and 2.1b, 0.16 on Figures 2.1c and 2.1d, 0.24 on Figures 2.1e and 2.1f), using the results from Appendix A.

On the one hand, the volatility distribution in the affine models from Table 2.1 (Schöbel-Zhu, Heston) has a short right tail ${ }^{7}$, while it has a more realistic long right tail in the non-affine models $(3 / 2$ model, Log-Normal, GARCH, and Inverse Gamma). Figures 2.1b, 2.1d and 2.1f (in log-scale), illustrate this difference between Heston and Inverse Gamma. The right tail of the Heston volatility decreases more quickly than that of the IGa volatility, therefore there is always a volatility level upon which the Heston volatility falls forever below the IGa one.

The left tail is also better described with non-affine models. For example, with the Heston model, the volatility can reach zero if the parameters $(\kappa, \theta, \lambda, \rho)$ do not satisfy the Feller condition $\left(2 \kappa \theta / \lambda^{2}>1\right)$. The effect of this condition on the left tail can be seen from Figures $2.1 \mathrm{a}$ and $2.1 \mathrm{~b}\left(2 \kappa \theta / \lambda^{2}=3.63\right)$ to Figures 2.1c and 2.1d $\left(2 \kappa \theta / \lambda^{2}=0.96\right)$ to Figures 2.1e and 2.1f $\left(2 \kappa \theta / \lambda^{2}=0.49\right)$. One can clearly see how the distribution piles up close to zero, to the point where zero becomes the most likely value for the volatility (Figure 2.1e). Unfortunately, the Feller condition is almost always violated in practice (Clark [2011], da Fonseca and Grasselli [2011], Ribeiro and Poulsen [2013], ...), which means that Figure 2.1e represents the normal behavior of the Heston model on real data.

\footnotetext{
${ }^{1}$ Schöbel and Zhu [1999]

${ }^{2}$ Heston [1993]

${ }^{3}$ Lewis [2000]

${ }^{4}$ Wiggins [1987]

${ }^{5} \mathrm{~A}$ more natural definition is $d S_{t}=\left(r_{d}-r_{f}\right) S_{t} d t+e^{V_{t}} S_{t} d W_{t}$ with $d V_{t}=\kappa\left(\theta-V_{t}\right) d t+\lambda d B_{t}$. See also Appendix B.

${ }^{6}$ Lewis [2000]

${ }^{7}$ using Rojo [1996]'s tail classification for example
} 


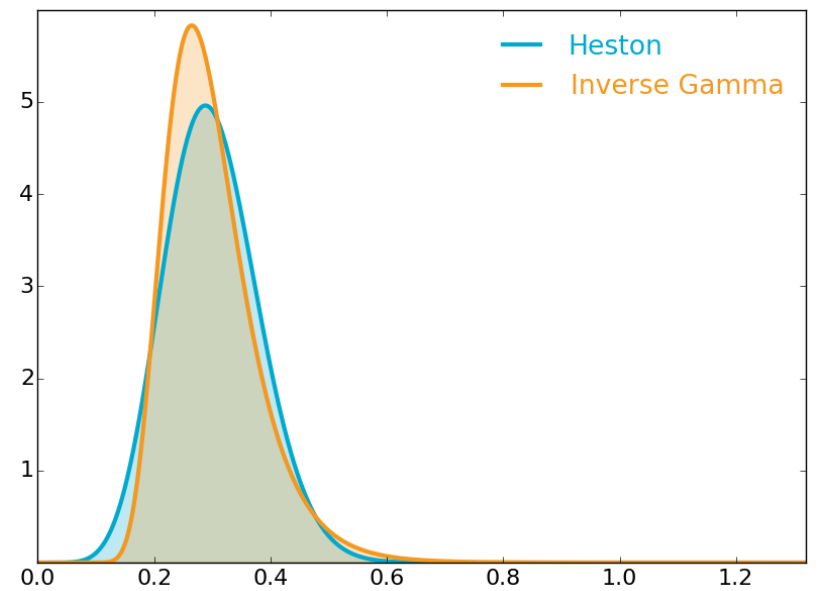

(a) mean $=0.30$, standard deviation $=0.08$

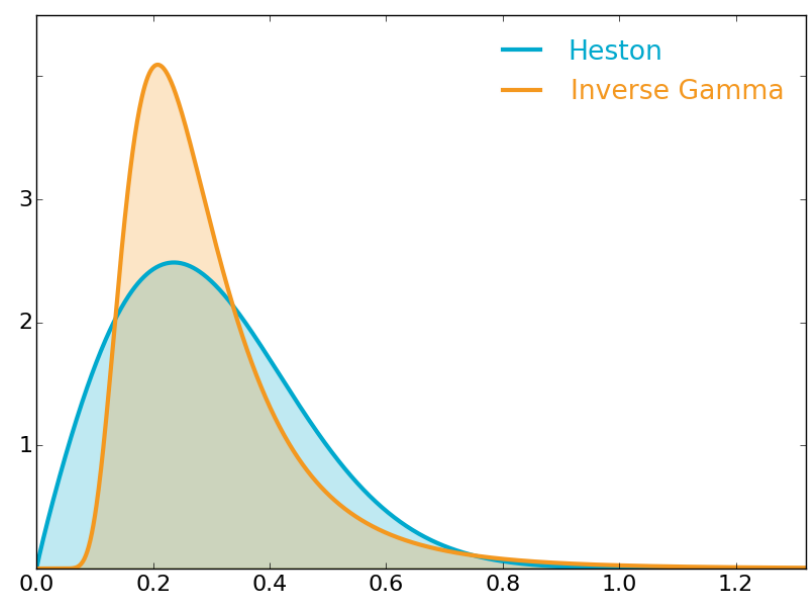

(c) mean $=0.30$, standard deviation $=0.16$

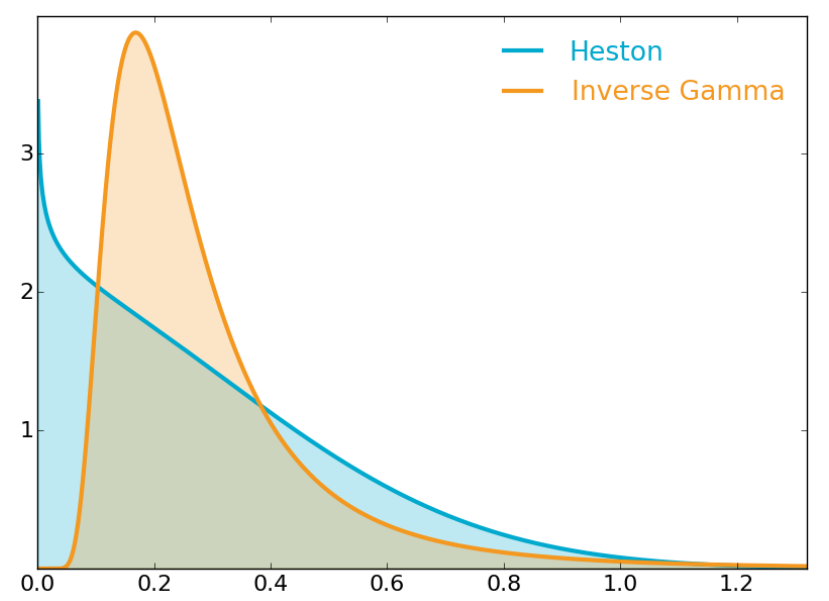

(e) mean $=0.30$, standard deviation $=0.24$

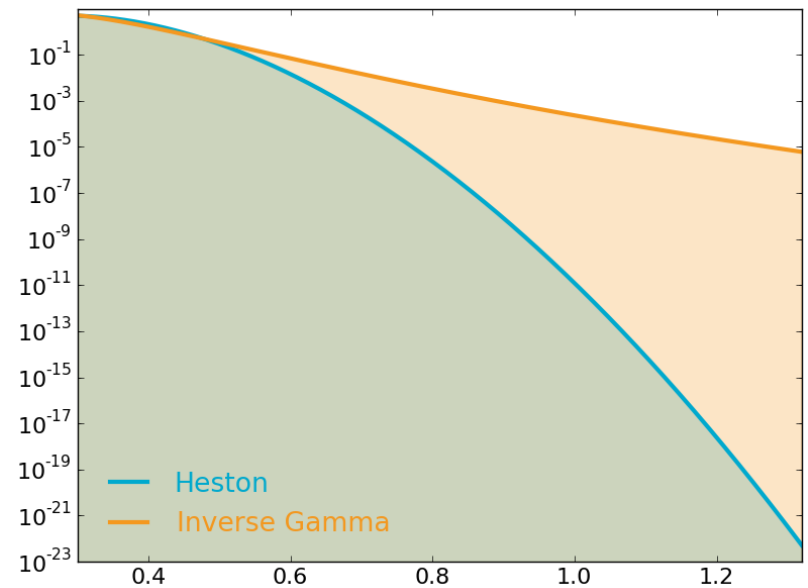

(b) mean $=0.30$, standard deviation $=0.08, \log$ scale

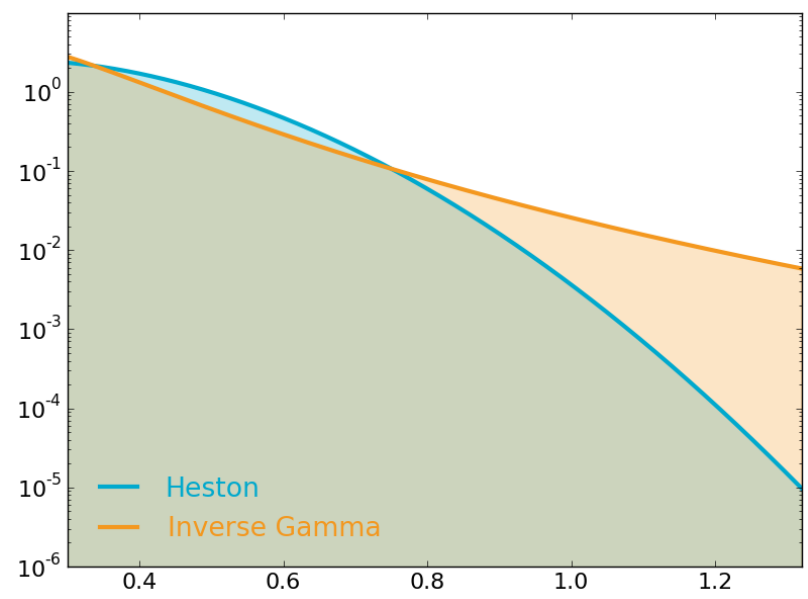

(d) mean $=0.30$, standard deviation $=0.16$, log scale

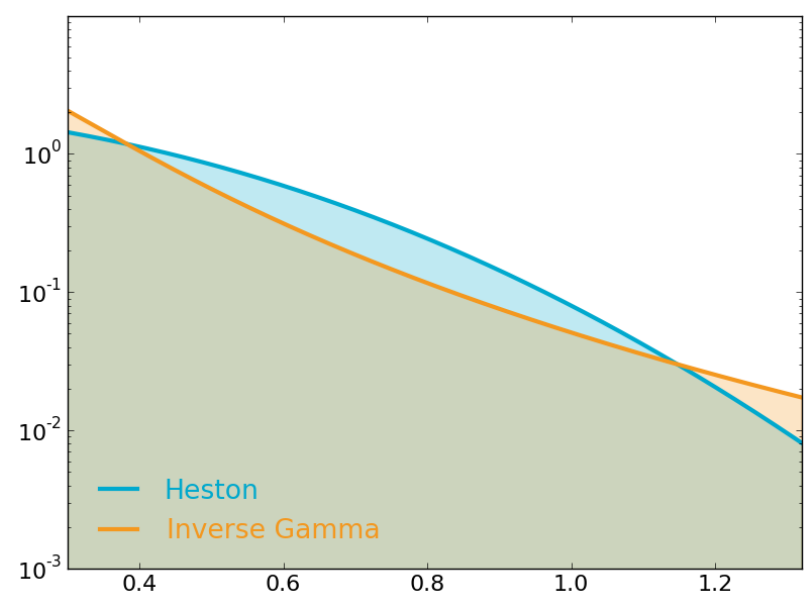

(f) mean $=0.30$, standard deviation $=0.24$, log scale

Figure 2.1: Volatility density 


\subsubsection{Empirical evidence}

Figure 2.1 strongly suggests that the IGa model 2.1, while being as parsimonious as Heston (four parameters $\kappa, \theta, \lambda, \rho)$, provides a much better description of realized and implied market volatility. Indeed, a number of empirical studies suggest that a non-affine stochastic volatility model of the type (2.1) compares favorably to other possible models, especially to affine models such as the Heston model. We can summarize these studies as listed below.

In equity markets:

- In Bouchaud and Potters [2003] (Chapter 7), an empirical analysis of the volatility of the S\&P 500 in the period 1990-2001 is performed. The distribution of the volatility is shown to be accurately fitted by two distributions: a log-normal distribution, and an inverse gamma distribution. Overall, the best fit is provided by the inverse gamma distribution, especially on the right tail of the volatility distribution (Figures 7.7 p.118 and 7.8 p.119). When discussing the shortcomings of the Heston stochastic volatility models, the authors explicitly say that "the empirical distribution of the volatility is closer to an inverse gamma distribution than to a gamma distribution" (p.143).

- Gander and Stephens [2007] test several possible distributions for the volatility (Tempered stable, Generalized Inverse Gaussian (including Gamma), Positive Hyperbolic, Inverse Gaussian and Inverse Gamma) on 14 stocks traded on the New York Stock Exchange. It is shown that the Inverse Gamma distribution provides the best fit for option pricing.

- Gatheral [2008] shows that the Double Lognormal stochastic volatility model (which contains (2.1) as a special case) fits SPX and VIX options much better than Double Heston, with stable parameters.

- Christoffersen et al. [2010] show, on S\&P 500 returns, VIX options and OTM S\&P 500 index option data, between 1996 and 2004, that the GARCH diffusion model (Inverse Gamma variance) significantly outperforms several other models including the Heston and the $3 / 2$ models. (They only consider the volatility parameterization (not variance) $d V_{t}=\left(\frac{\kappa \theta}{2} V_{t}^{2 a-1}-\frac{\kappa}{2} V_{t}^{2 a+1}-\frac{\lambda^{2}}{8} V_{t}^{4 b-3}\right) d t+$ $\frac{\lambda}{2} V_{t}^{2 b-1} d B_{t}$, which does not contain the Inverse Gamma model.)

- Furthermore, Kaeck and Alexander [2012] show that allowing for non-affine dynamics (like (2.1)) is more important than the inclusion of jumps. In particular, augmenting an affine model such as the Heston model with jumps leads to a stochastic volatility model that is still significantly inferior to a more parsimonious non-affine model like GARCH without jumps.

- Finally, Ma and Serota [2014] analyze the volatility of the S\&P 100, S\&P 500 and DJIA, as well as the VIX index between 1990 and 2014. They show that the Generalized Inverse Gamma distribution (which contain the Inverse Gamma distribution) fits volatility best.

In fixed-income markets:

- Fornari and Mele [2001] calibrate the Power Arch model to futures contracts on the Italian 10-year government bond, between 1991 to 1997. The power $p$ is estimated on three different subsamples. The results, $0.86,0.99$ and 1.19 , are very close to an IGa volatility $(p=1)$. In particular, the GARCH diffusion model $(p=2)$ is rejected.

- Fornari and Mele [2006] then study a slightly different stochastic volatility model, of the type $d V_{t}^{p}=\kappa\left(\theta-V_{t}^{p}\right) d t+\lambda V_{t}^{\eta p} d B_{t}$, with two parameters $p$ and $\eta$. They fit these two parameters to weekly 3 -month US Treasury bills rates between 1973 and 1995. Their estimates, $\hat{p}=1.0326$ and $\hat{\eta}=1.0014$, are statistically indistinguishable from 1 (Inverse Gamma volatility). Remark that the volatility term for the asset $S$ (which is an interest rate in there case) is of the form $V_{t} \sqrt{S_{t}} d W_{t}$, which is different from (2.1). However, they state that a volatility of the form $V_{t}\left|S_{t}\right|^{d} d W_{t}, d \geq 0.5$ (which contains $(2.1)$ for $d=1$ ), would not dramatically change their empirical results. 
Beyond better calibration and more realistic volatility distribution and volatility paths, a model such as 2.1 generates a more realistic dynamics for the implied volatility surface (Tataru and Fisher [2012]), which reduces the tracking volatility in hedging P\&Ls (Sepp [2015]), and drastically improves portfolio allocation (Hansis [2010]).

\section{Closed-form expansion for fast option pricing}

In the previous section, a number of reasons have been documented in favor of a non-affine Inverse Gamma stochastic volatility model over affine models. However, the main reason why affine models are used in practice is not their realism but their tractability. Indeed their Fourier (or Laplace) transform is available in closed form, which makes pricing possible by inverse transform. Non-affine models such as the IGa model do not have a closed form solution for their Fourier transform, which makes them $a$ priori less tractable.

In Sepp [2014], an affine moment-matching approximation of the moment generation function for (2.1) is proposed, making pricing possible by inverse transform. The advantage of this approach is that jumps can be factored in during the matching as well. Here we propose a more straightforward approach, namely a closed-form volatility-of-volatility expansion for vanilla options prices. Compared to Sepp [2014], the main advantages of our approach are that:

- It is simpler. If an approximation is to be made, it is more straightforward and intuitive to approximate the price directly rather than a transform of the price. This also makes pricing and subsequent calibration much faster.

- No moment matching is required. Firstly, matching moments with an affine model can create unexpected problems (recall Figure 2.1e). Secondly, for most stochastic volatility models, moments higher than 1 cease to exist for large maturities (Andersen and Piterbarg [2007]). In Sepp [2014], the author advises against going beyond a second-order approximation, as only 5 moments were shown to exist. Our approach does not have such limitation on the order of approximation.

- Importantly, our approximation approach is naturally suitable for time-dependent parameters.

In the following subsection 3.1, we provide a closed-form expansion for the price of a European put option under Inverse Gamma volatility (2.1) with time-dependent parameters (equation (3.1)). The method is based on the methodology developed in Benhamou et al. [2010], adapted to the Inverse Gamma model and extended to time-dependent $\kappa$. In Benhamou et al. [2010], the closed-form volatility of volatility expansion methodology was applied to the Heston stochastic volatility with time-dependent parameters. It was shown to be very accurate, and much faster than Fourier methods.

The coefficients of the closed-form expansion are explicitly given by time integrals of the (time-dependent) parameters (equation (3.5)). Importantly, any shape for the time evolution of the parameters can be handled. In practice though, piecewise-constant parameters can be seen as a good compromise between richness and tractability. Thus, we present generic recusions formulas for the coefficients (3.5) when the model parameters are piecewise constant. These generic recursions are easy to implement, and can be used for expansion coefficients to any order.

Finally, in order to compare our prices to another method, and as transform methods are not available, we explain how to implement an efficient Monte Carlo scheme to price options under IGa volatility. 


\subsection{Closed-form expansion}

Theorem 3.1. The second-order expansion for the price $P_{I G a}=P_{I G a}\left(S_{0}, K, T, r_{d}, r_{f} ; \kappa, \theta, \lambda, \rho\right)$ of a European put option with Inverse Gamma volatility is explicitly given by

$$
P_{I G a}=P_{B S}\left(x_{0}, \psi_{T}\right)+\sum_{i=0}^{2} a_{i, T} \frac{\partial^{i+1}}{\partial x^{i} y} P_{B S}\left(x_{0}, \psi_{T}\right)+\sum_{i=0}^{1} b_{2 i, T} \frac{\partial^{2 i+2}}{\partial x^{2 i} y^{2}} P_{B S}\left(x_{0}, \psi_{T}\right)+\mathcal{E}
$$

with

$$
\begin{aligned}
x_{0} & =\log \left(S_{0}\right) \\
v_{0, t} & =e^{-\int_{0}^{t} \kappa_{z} d z}\left(v_{0}+\int_{0}^{t} \kappa_{s} \theta_{s} e^{\int_{0}^{s} \kappa_{z} d z} d s\right) \\
\psi_{T} & =\int_{0}^{T} v_{0, t}^{2} d t \\
a_{0, T} & =\int_{0}^{T} e^{\int_{0}^{s} 2 \kappa_{z} d z} \lambda_{s}^{2} v_{0, s}^{2} d s \int_{s}^{T} e^{-\int_{0}^{t} 2 \kappa_{z} d z} d t \\
a_{1, T} & =2 \int_{0}^{T} e^{\int_{0}^{s} \kappa_{z} d z} \rho_{s} \lambda_{s} v_{0, s}^{2} d s \int_{s}^{T} e^{-\int_{0}^{t} \kappa_{z} d z} v_{0, t} d t \\
a_{2, T} & =2 \int_{0}^{T} e^{\int_{0}^{s} \kappa_{z} d z} \rho_{s} \lambda_{s} v_{0, s}^{2} d s \int_{s}^{T} 2 \rho_{t} \lambda_{t} v_{0, t} d t \int_{t}^{T} e^{-\int_{0}^{u} \kappa_{z} d z} v_{0, u} d u \\
& +2 \int_{0}^{T} e^{\int_{0}^{s} \kappa_{z} d z} \rho_{s} \lambda_{s} v_{0, s}^{2} d s \int_{s}^{T} e^{\int_{0}^{t} \kappa_{z} d z} \rho_{t} \lambda_{t} v_{0, t}^{2} d t \int_{t}^{T} e^{-\int_{0}^{u} 2 \kappa_{z} d z} d u \\
b_{0, T} & =4 \int_{0}^{T} e^{\int_{0}^{s} 2 \kappa_{z} d z} \lambda_{s}^{2} v_{0, s}^{2} d s \int_{s}^{T} e^{-\int_{0}^{t} \kappa_{z} d z} v_{0, t} d t \int_{t}^{T} e^{-\int_{0}^{u} \kappa_{z} d z} v_{0, u} d u \\
b_{2, T} & =\frac{a_{1, T}^{2}}{2}
\end{aligned}
$$

where $P_{B S}(x, y)=P_{B S}\left(x, y ; K, T, r_{d}, r_{f}\right)$ is the Black-Scholes put price with spot $e^{x}$ and integrated variance $y$,

$$
\begin{aligned}
P_{B S}(x, y)= & K e^{-\int_{0}^{T} r_{d}(t) d t} \mathcal{N}\left(\frac{1}{\sqrt{y}} \log \left(\frac{K e^{-\int_{0}^{T} r_{d}(t) d t}}{e^{x} e^{-\int_{0}^{T} r_{q}(t) d t}}\right)+\frac{1}{2} \sqrt{y}\right) \\
& -e^{x} e^{-\int_{0}^{T} r_{q}(t) d t} \mathcal{N}\left(\frac{1}{\sqrt{y}} \log \left(\frac{K e^{-\int_{0}^{T} r_{d}(t) d t}}{e^{x} e^{-\int_{0}^{T} r_{q}(t) d t}}\right)-\frac{1}{2} \sqrt{y}\right),
\end{aligned}
$$

and $\mathcal{E}$ is the error term of the second-order expansion.

Proof. The proof or expansion (3.1) is available in Appendix C. It is based on the proof of the Heston expansion in Benhamou et al. [2010], that we extended to non-constant $\kappa$, and adapted to the IGa model.

Remark 3.1. One can also easily obtain closed-form expansion for the Greeks by adapting the proof of Theorem 3.1 to it. More generally the methodology adopted in Theorem 3.1 can be adapted to any options that have closed-form Black-Scholes prices with time dependent parameters, for example barrier options (Lo et al. [2003], Rapisarda [2003]). 


\subsection{Recursions for expansion coefficients}

The above coefficients (3.5) are expressed for general deterministic parameters $\left(\kappa_{t}, \theta_{t}, \lambda_{t}, \rho_{t}\right)_{0 \leq t \leq T}$. In this subsection, we propose an explicit recursive algorithm to compute the coefficients when the parameters are piecewise constant.

Let $T_{0}=0<T_{1}<T_{2}<\cdots<T_{N}=T$ be a partition of $[0, T]$. We now suppose that the parameters are constant on each interval:

$$
\left(\kappa_{t}, \theta_{t}, \lambda_{t}, \rho_{t}\right) \quad:=\left(\kappa_{i}, \theta_{i}, \lambda_{i}, \rho_{i}\right) \quad \forall t \in\left[T_{i}, T_{i+1}[\right.
$$

\subsubsection{Integral operator}

Firstly, we define recursively the following integral operator ${ }^{1}$

$$
\begin{gathered}
\omega_{t, T}^{(\kappa, l)}=\int_{t}^{T} e^{\int_{0}^{u} \kappa_{z} d z} l_{u} d u \quad \forall t \in[0, T] \\
\omega_{t, T}^{\left(\kappa_{n}, l_{n}\right), \ldots,\left(\kappa_{1}, l_{1}\right)}=\omega_{t, T}^{\left(\kappa_{n}, l_{n} \omega_{., T}^{\left(\kappa_{n-1}, l_{n-1}\right), \ldots,\left(\kappa_{1}, l_{1}\right)}\right)} \quad \forall t \in[0, T]
\end{gathered}
$$

Using this notation, the coefficients from the price expansion 3.1 can be expressed as follows:

$$
\begin{aligned}
\psi_{T} & =\omega_{0, T}^{\left(0, v_{0, .}^{2}\right)} \\
a_{0, T} & =\omega_{0, T}^{\left(2 \kappa, \lambda^{2} v_{0, .}^{2}\right),(-2 \kappa, 1)} \\
a_{1, T} & =2 \omega_{0, T}^{\left(\kappa, \rho \lambda v_{0, .}^{2}\right),\left(-\kappa, v_{0, .}\right)} \\
a_{2, T} & =2 \omega_{0, T}^{\left(\kappa, \rho \lambda v_{0, .}^{2}\right),\left(0,2 \rho \lambda v_{0, .}\right),\left(-\kappa, v_{0, .}\right)}+2 \omega_{0, T}^{\left(\kappa, \rho \lambda v_{0, .}^{2}\right),\left(\kappa, \rho \lambda v_{0, .}^{2}\right),(-2 \kappa, 1)} \\
b_{0, T} & =4 \omega_{0, T}^{\left(2 \kappa, \lambda^{2} v_{0, .}^{2}\right),\left(-\kappa, v_{0, .}\right),\left(-\kappa, v_{0, .}\right)}
\end{aligned}
$$

\subsubsection{Recursions}

Let $l_{1}, l_{2}, l_{3}, \ldots$ be deterministic functions. When these functions are piecewise constant,

$$
l_{k}(t):=l_{k, i}, t \in\left[T_{i}, T_{i+1}[, k=1,2, \ldots,\right.
$$

then the following integral operators at time $T_{i+1}$ can be expressed as a function of the integral operators at time $T_{i}$ :

$$
\begin{aligned}
\omega_{0, T_{i+1}}^{\left(n_{1} \kappa, l_{1} v_{0, .}^{p_{1}}\right)} & =\omega_{0, T_{i}}^{\left(n_{1} \kappa, l_{1} v_{0, .}^{p_{1}}\right)}+e_{0, T_{i}}^{n_{1}} l_{1, i} \varphi_{T_{i}, T_{i+1}}^{\left(n_{1}, 0, p_{1}\right)} \\
\omega_{0, T_{i+1}}^{\left(n_{2} \kappa, l_{2} v_{0, .}^{p_{2}}\right),\left(n_{1} \kappa, l_{1} v_{0, .}^{p_{1}}\right)} & =\omega_{0, T_{i}}^{\left(n_{2} \kappa, l_{2} v_{0, .}^{p_{2}}\right),\left(n_{1} \kappa, l_{1} v_{0 . .}^{p_{1}}\right)} \\
& +\omega_{0, T_{i}}^{\left(n_{2} \kappa, l_{2} v_{0, .}^{p_{2}}\right)} e_{0, T_{i}}^{n_{1}} l_{1, i} \varphi_{T_{i}, T_{i+1}}^{\left(n_{1}, 0, p_{1}\right)} \\
& +e_{0, T_{i}}^{n_{2}+n_{1}} l_{2, i} l_{1, i} \varphi_{T_{i}, T_{i+1}}^{\left(n_{2}, 0, p_{2}\right),\left(n_{1}, 0, p_{1}\right)}
\end{aligned}
$$

\footnotetext{
${ }^{1}$ which corresponds to Def. 5.1 in Benhamou et al. [2010] extended to non-constant $\kappa$
} 


$$
\begin{aligned}
\omega_{0, T_{i+1}}^{\left(n_{3} \kappa, l_{3} v_{0, .}^{p_{3}}\right),\left(n_{2} \kappa, l_{2} v_{0, .}^{p_{2}}\right),\left(n_{1} \kappa, l_{1} v_{0, .}^{p_{1}}\right)} & =\omega_{0, T_{i}}^{\left(n_{3} \kappa, l_{3} v_{0, .}^{p_{3}}\right),\left(n_{2} \kappa, l_{2} v_{0, .}^{p_{2}}\right),\left(n_{1} \kappa, l_{1} v_{0, .}^{p_{1}}\right)} \\
& +\omega_{0, T_{i}}^{\left(n_{3} \kappa, l_{3} v_{0, .}^{p_{3}}\right),\left(n_{2} \kappa, l_{2} v_{0, .}^{p_{2}}\right)} e_{0, T_{i}}^{n_{1}} l_{1, i} \varphi_{T_{i}, T_{i+1}}^{\left(n_{1}, 0, p_{1}\right)} \\
& +\omega_{0, T_{i}}^{\left(n_{3} \kappa, l_{3} v_{0 . .}^{p_{3}}\right)} e_{0, T_{i}}^{n_{2}+n_{1}} l_{2, i} l_{1, i} \varphi_{T_{i}, T_{i+1}}^{\left(n_{2}, 0, p_{2}\right),\left(n_{1}, 0, p_{1}\right)} \\
& +e_{0, T_{i}}^{n_{3}+n_{2}+n_{1}} l_{3, i} l_{2, i} l_{1, i} \varphi_{T_{i}, T_{i+1}}^{\left(n_{3}, 0, p_{3}\right),\left(n_{2}, 0, p_{2}\right),\left(n_{1}, 0, p_{1}\right)}
\end{aligned}
$$

and so on, where $n_{1}, n_{2}, n_{3}, \ldots$ and $p_{1}, p_{2}, p_{3}, \ldots$ are integers, $e_{0, t}=e^{\int_{0}^{t} \kappa_{z} d z}$, and for all $T_{i} \leq t \leq T_{i+1}$, $\varphi$ is defined as follows:

$$
\begin{aligned}
\varphi_{t, T_{i+1}}^{\left(n_{1}, m_{1}, p_{1}\right)} & =\int_{t}^{T_{i+1}} e^{n_{1} \int_{T_{i}}^{s} \kappa_{z} d z} \gamma(s)^{m_{1}} v_{0, s}^{p_{1}} d s=\int_{t}^{T_{i+1}} e^{n_{1} \kappa_{i} \Delta T_{i} \gamma(s)} \gamma(s)^{m_{1}} v_{0, s}^{p_{1}} d s \\
\varphi_{t, T_{i+1}}^{\left(n_{k}, m_{k}, p_{k}\right), \cdots,\left(n_{1}, m_{1}, p_{1}\right)} & =\int_{t}^{T_{i+1}} e^{n_{k} \int_{T_{i}}^{s} \kappa_{z} d z} \gamma(s)^{m_{k}} v_{0, s}^{p_{k}} \varphi_{t, T_{i+1}}^{\left(n_{k-1}, m_{k-1}, p_{k-1}\right), \cdots,\left(n_{1}, m_{1}, p_{1}\right)} d s \\
& =\int_{t}^{T_{i+1}} e^{n_{k} \kappa_{i} \Delta T_{i} \gamma(s)} \gamma(s)^{m_{k}} v_{0, s}^{p_{k}} \varphi_{t, T_{i+1}}^{\left(n_{k-1}, m_{k-1}, p_{k-1}\right), \cdots,\left(n_{1}, m_{1}, p_{1}\right)} d s
\end{aligned}
$$

where $\gamma(s)=\frac{s-T_{i}}{\Delta T_{i}}$ with $\Delta T_{i}=T_{i+1}-T_{i}$, and $n_{1}, m_{1}, p_{1}, \ldots, n_{k}, m_{k}, p_{k}$ are integers.

It should be noted that when the parameters are piecewise constant (equation (3.7)), $\varphi$ can be computed explicitly by recursion. Define $\Delta \kappa_{i}=\kappa_{i+1}-\kappa_{i}, \Delta \theta_{i}=\theta_{i+1}-\theta_{i}, \Delta \lambda_{i}=\lambda_{i+1}-\lambda_{i}$ and $\Delta \rho_{i}=\rho_{i+1}-\rho_{i}$, and let $t \in\left[T_{i}, T_{i+1}\right]$. Then

$$
v_{0, t}=\theta_{i}+\left(v_{0, T_{i}}-\theta_{i}\right) e^{-\kappa_{i} \Delta T_{i} \gamma(t)}
$$

and, using the definition of $\varphi$, basic integration and integration by parts, the following recursions hold

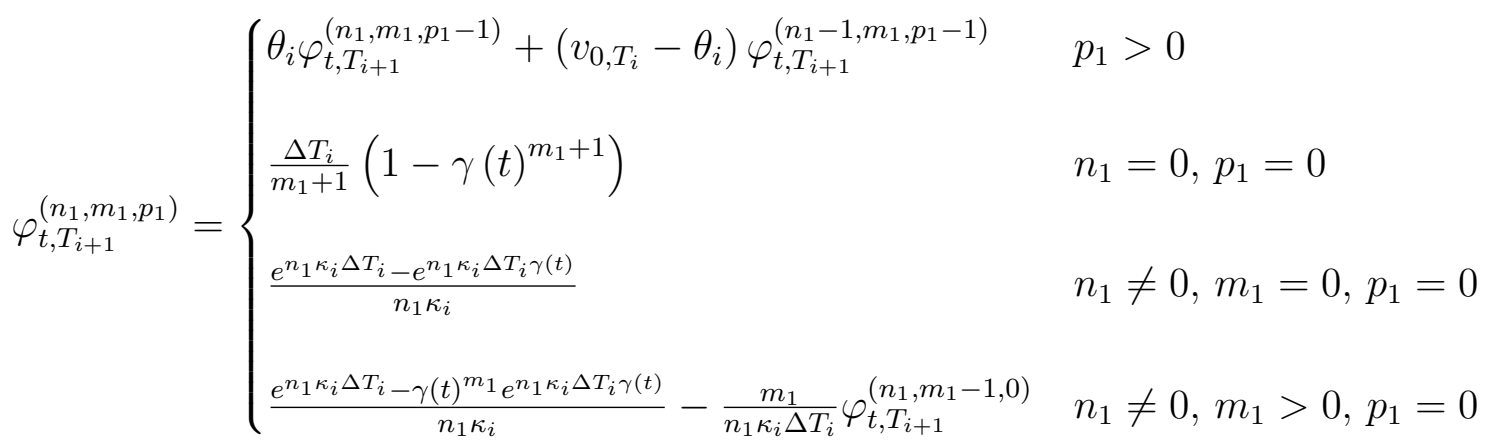

and for every integer $k>1$ : 


$$
\begin{aligned}
& \varphi_{t, T_{i+1}}^{\left(n_{k}, m_{k}, p_{k}\right),\left(n_{k-1}, m_{k-1}, p_{k-1}\right), \cdots,\left(n_{1}, m_{1}, p_{1}\right)}= \\
& \begin{cases}\theta_{i} \varphi_{t, T_{i+1}}^{\left(n_{k}, m_{k}, p_{k}-1\right), \cdots}+\left(v_{0, T_{i}}-\theta_{i}\right) \varphi_{t, T_{i+1}}^{\left(n_{k}-1, m_{k}, p_{k}-1\right)} & p_{k}>0 \\
-\frac{\Delta T_{i}}{m_{k}+1} \gamma(t)^{m_{k}+1} \varphi_{t, T_{i+1}}^{\left(n_{k-1}, m_{k-1}, p_{k-1}\right), \cdots}+\frac{\Delta T_{i}}{m_{k}+1} \varphi_{t, T_{i+1}}^{\left(n_{k-1}, m_{k}+m_{k-1}+1, p_{k-1}\right), \cdots} & n_{k}=0, p_{k}=0 \\
-\frac{e^{n_{k} \kappa_{i} \Delta T_{i} \gamma(t)}}{n_{k} \kappa_{i}} \varphi_{t, T_{i+1}}^{\left(n_{k-1}, m_{k-1}, p_{k-1}\right), \cdots}+\frac{1}{n_{k} \kappa_{i}} \varphi_{t, T_{i+1}}^{\left(n_{k}+n_{k-1}, m_{k-1}, p_{k-1}\right), \cdots} & n_{k} \neq 0, m_{k}=0, p_{k}=0 \\
-\frac{e^{n_{k} \kappa_{i} \Delta T_{i} \gamma(t)}}{n_{k} \kappa_{i}}\left[\sum_{j=0}^{m_{k}} \gamma(t)^{j} \frac{m_{k} !}{j !}\left(\frac{-1}{n_{k} \kappa_{i} \Delta T_{i}}\right)^{m_{k}-j}\right] \varphi_{t, T_{i+1}}^{\left(n_{k-1}, m_{k-1}, p_{k-1}\right), \cdots} & \\
+\frac{1}{n_{k} \kappa_{i}} \sum_{j=0}^{m_{k}} \frac{m_{k} !}{j !}\left(\frac{-1}{n_{k} \kappa_{i} \Delta T_{i}}\right)^{m_{k}-j} \varphi_{t, T_{i+1}}^{\left(n_{k}+n_{k-1}, m_{k-1}+j, p_{k-1}\right), \cdots} & n_{k} \neq 0, m_{k}>0, p_{k}=0\end{cases}
\end{aligned}
$$

All these equation are sufficient to compute $\varphi_{T_{i}, T_{i+1}}^{\left(n_{k}, m_{k}, p_{k}\right), \cdots,\left(n_{1}, m_{1}, p_{1}\right)}$ for any integers $\left(n_{1}, m_{1}, p_{1}\right), \cdots,\left(n_{k}, m_{k}, p_{k}\right)$, making it possible to implement the integral recursions (3.11), (3.12) and (3.13), which, in turn, make it possible to compute explicitly the expansion coefficients (3.10) when the parameters of the stochastic volatility model are piecewise constant.

\subsection{Monte Carlo}

An obvious alternative method for computing the price of a European put option under the IGa stochastic volatility model is the Monte Carlo method. Using the expression

$$
P_{I G a}\left(x_{0}, v_{0}\right)=\mathbb{E}\left[P_{B S}\left(x_{0}+\int_{0}^{T} \rho_{t} V_{t} d B_{t}-\frac{1}{2} \int_{0}^{T}\left(\rho_{t} V_{t}\right)^{2} d t, \int_{0}^{T}\left(1-\rho_{t}^{2}\right) V_{t}^{2} d t\right)\right],
$$

(cf. equation (C.4)), only the simulation of the volatility is needed. Then, one can take advantage of the strong solution of the Inverse Gamma diffusion (Zhao [2009])

$$
V_{t}=\frac{1}{Z_{t}}\left(V_{0}+\int_{0}^{t} \kappa_{s} \theta_{s} Z_{s} d s\right)
$$

where $Z$ is a geometric Brownian motion

$$
d Z_{t}=\left(\kappa_{t}+\lambda_{t}^{2}\right) Z_{t} d t-\lambda_{t} Z_{t} d B_{t}
$$

ie.

$$
Z_{t}=\exp \left(\int_{0}^{t}\left(\kappa_{s}+\frac{1}{2} \lambda_{s}^{2}\right) d s-\int_{0}^{t} \lambda_{s} d B_{s}\right)
$$

to derive the following unconditionally stable discretization scheme (called "Pathwise Adapted Linearization" in Kahl and Jackel [2006])

$$
\begin{aligned}
\delta_{n} & \leftarrow\left(\kappa_{t_{n}}+\frac{1}{2} \lambda_{t_{n}}^{2}\right) \Delta t_{n}-\lambda_{t_{n}} \Delta B_{n} \\
V_{t_{n+1}} & \leftarrow V_{t_{n}} e^{-\delta_{n}}+\kappa_{t_{n}} \theta_{t_{n}} \frac{1-e^{-\delta_{n}}}{\delta_{n}} \Delta t_{n}
\end{aligned}
$$

where $0=t_{0} \leq \ldots \leq t_{n} \leq \ldots \leq t_{N}=T$ is a time discretization of the interval $[0, T]$, with $\Delta t_{n}:=$ $t_{n+1}-t_{n}$ and $\Delta B_{n}:=B_{t_{n+1}}-B_{t_{n}}$. This scheme ensures in particular that the paths of $V$ remain positive. 


\section{Numerical experiments}

This section provides numerical tests of the fast pricing method proposed in this paper. The Inverse Gamma stochastic volatility model will be calibrated to market data using the pricing formula (3.1). Three foreign exchange data sets will be used, detailed in subsection 4.1. For each test case, we will provide the calibration errors and expansion errors for implied volatility surfaces (Subsection 4.2).

\subsection{Datasets}

Three full sets of foreign exchange market data are provided here for easy benchmarking. We provide the strikes used for the implied volatility surface, and the equivalent constant rates for each maturity (the constant rate $r_{e q}(T)$ equivalent to a time-dependent rate $r(t), 0 \leq t \leq T$ is defined by $r_{e q}(T):=$ $\left.\frac{1}{T} \int_{0}^{T} r(t) d t\right)$. The corresponding market implied volatility surfaces will be provided in subsection 4.2 (Tables 4.4, 4.5 and 4.6), along with the corresponding calibration errors and expansion errors. All the numbers quoted here have been rounded.

\subsubsection{Set 1: AUD/USD 17 June $2014\left(S_{0}=0.9335\right)$}

\begin{tabular}{llllll}
\multicolumn{2}{l}{ Strikes } \\
\hline Mat & 10 Put & 25 Put & ATM & 25 Call & 10 Call \\
\hline $1 \mathrm{M}$ & 0.9103 & 0.9233 & 0.9356 & 0.9469 & 0.9572 \\
$3 \mathrm{M}$ & 0.8906 & 0.9168 & 0.9401 & 0.9605 & 0.9795 \\
$6 \mathrm{M}$ & 0.8664 & 0.9100 & 0.9469 & 0.9780 & 1.0078 \\
$1 \mathrm{Y}$ & 0.8322 & 0.9027 & 0.9609 & 1.0096 & 1.0580 \\
\hline
\end{tabular}

\begin{tabular}{lll}
\multicolumn{2}{l}{ Rates } & \\
\hline Mat & foreign & domestic \\
\hline $1 \mathrm{M}$ & $2.80 \%$ & $0.21 \%$ \\
$3 \mathrm{M}$ & $2.89 \%$ & $0.31 \%$ \\
$6 \mathrm{M}$ & $3.03 \%$ & $0.45 \%$ \\
$1 \mathrm{Y}$ & $3.26 \%$ & $0.69 \%$ \\
\hline
\end{tabular}

Table 4.1: Market data, AUDUSD, 17 June 2014

\subsubsection{Set 2: USD/JPY 11 June $2014\left(S_{0}=102.00\right)$}

\begin{tabular}{llrlll}
\multicolumn{2}{l}{ Strikes } \\
\hline Mat & 10 Put & 25 Put & ATM & 25 Call & 10 Call \\
\hline $1 \mathrm{M}$ & 99.78 & 100.88 & 101.99 & 103.09 & 104.16 \\
$3 \mathrm{M}$ & 97.47 & 99.77 & 101.98 & 104.15 & 106.31 \\
$6 \mathrm{M}$ & 94.75 & 98.47 & 102.00 & 105.46 & 109.06 \\
$1 \mathrm{Y}$ & 90.04 & 96.34 & 102.01 & 107.67 & 114.06 \\
\hline
\end{tabular}

\begin{tabular}{llc}
\multicolumn{3}{l}{ Rates } \\
\hline Mat & foreign & domestic \\
\hline $1 \mathrm{M}$ & $0.20 \%$ & $-0.04 \%$ \\
$3 \mathrm{M}$ & $0.29 \%$ & $0.07 \%$ \\
$6 \mathrm{M}$ & $0.40 \%$ & $0.16 \%$ \\
$1 \mathrm{Y}$ & $0.52 \%$ & $0.21 \%$ \\
\hline
\end{tabular}

Table 4.2: Market data, USDJPY, 11 June 2014

\subsubsection{Set 3: USD/SGD 04 September $2014\left(S_{0}=1.2541\right)$}

\begin{tabular}{llllll}
\multicolumn{2}{l}{ Strikes } \\
\hline Mat & 10 Put & 25 Put & ATM & 25 Call & 10 Call \\
\hline $1 \mathrm{M}$ & 1.2397 & 1.2466 & 1.2542 & 1.2637 & 1.2755 \\
$2 \mathrm{M}$ & 1.2334 & 1.2432 & 1.2542 & 1.2688 & 1.2871 \\
$3 \mathrm{M}$ & 1.2286 & 1.2406 & 1.2543 & 1.2729 & 1.2970 \\
$6 \mathrm{M}$ & 1.2152 & 1.2339 & 1.2545 & 1.2836 & 1.3233 \\
$1 \mathrm{Y}$ & 1.1945 & 1.2232 & 1.2548 & 1.3018 & 1.3704 \\
\hline
\end{tabular}

\begin{tabular}{lll}
\multicolumn{3}{l}{ Rates } \\
\hline Mat & foreign & domestic \\
\hline $1 \mathrm{M}$ & $0.16 \%$ & $0.17 \%$ \\
$2 \mathrm{M}$ & $0.19 \%$ & $0.19 \%$ \\
$3 \mathrm{M}$ & $0.28 \%$ & $0.27 \%$ \\
$6 \mathrm{M}$ & $0.48 \%$ & $0.47 \%$ \\
$1 \mathrm{Y}$ & $0.61 \%$ & $0.57 \%$ \\
\hline
\end{tabular}

Table 4.3: Market data, USDSGD, 04 September 2014 
Our empirical analyses suggest that these three data sets are representative of the behavior on market data of the model and of the expansion. In particular, our empirical findings will be similar for each data set, leading us to present only three examples, as providing more examples would not provide much additional information.

\subsection{Calibration}

Using the closed-form expansion (3.1), we can respectively calibrate the Inverse Gamma model to the three market data sets. The four (piecewise-constant) stochastic parameters $\kappa, \theta, \lambda$ and $\rho$ are calibrated, along with the initial volatility $V_{0}$. This calibration process can be assessed from the implied volatility calibration error. Then, using these parameters, we will estimate the expansion error, by comparing the implied volatility provided by the expansion (3.1) to a Monte Carlo price (Subsection 3.3), computed with 24 time steps per day and $M=1000000$ paths (to keep both bias and variance very low).

\subsubsection{Set 1: AUD/USD 17 June $2014\left(S_{0}=0.9335\right)$}

The estimated initial volatility is $V_{0}=6.49 \%$, the estimated piecewise-constant stochastic parameters are given by

\begin{tabular}{llrll} 
& $\kappa$ & \multicolumn{1}{c}{$\theta$} & \multicolumn{1}{c}{$\lambda$} & \multicolumn{1}{c}{$\rho$} \\
\hline $1 \mathrm{M}$ & 4.19 & $6.39 \%$ & 1.71 & -0.40 \\
$3 \mathrm{M}$ & 2.33 & $11.01 \%$ & 1.12 & -0.74 \\
$6 \mathrm{M}$ & 2.26 & $11.85 \%$ & 1.25 & -0.73 \\
$1 \mathrm{Y}$ & 1.80 & $12.52 \%$ & 0.87 & -0.92 \\
\hline
\end{tabular}

and the calibration and expansion errors are given below (rounded to the nearest basis point):

\begin{tabular}{llllll} 
& \multicolumn{1}{l}{$10 \mathrm{Put}$} & $25 \mathrm{Put}$ & ATM & 25 Call & 10 Call \\
\hline $1 \mathrm{M}$ & $7.48[-0.04][-0.04]$ & $6.87[-0.02][-0.01]$ & $6.38[0.06][0.00]$ & $6.19[0.00][0.02]$ & $6.19[-0.03][-0.00]$ \\
$3 \mathrm{M}$ & $8.46[0.05][-0.12]$ & $7.48[-0.05][-0.03]$ & $6.68[0.03][0.03]$ & $6.36[-0.04][0.08]$ & $6.39[0.05][-0.08]$ \\
$6 \mathrm{M}$ & $9.93[0.02][-0.15]$ & $8.43[-0.03][-0.03]$ & $7.30[0.06][0.04]$ & $6.82[-0.07][0.13]$ & $6.90[0.08][-0.29]$ \\
$1 \mathrm{Y}$ & $11.51[-0.05][-0.19]$ & $9.53[0.00][-0.04]$ & $8.05[0.16][0.07]$ & $7.47[-0.14][0.19]$ & $7.57[0.15][-0.63]$ \\
\hline
\end{tabular}

Table 4.4: AUDUSD Market implied volatility [calibration error] [expansion error] in \%

The median absolute deviation of the calibration error is 5.0bp, and its mean absolute deviation is $5.7 \mathrm{bp}$. The median absolute deviation of the expansion error is $5.5 \mathrm{bp}$, and its mean absolute deviation is $10.9 \mathrm{bp}$. Overall, the median absolute deviation of the total error is $6.0 \mathrm{bp}$, and its mean absolute deviation is $10.5 \mathrm{bp}$.

\subsubsection{Set 2: USD/JPY 11 June $2014\left(S_{0}=102.00\right)$}

The estimated initial volatility is $V_{0}=4.42 \%$, the estimated piecewise-constant stochastic parameters are given by 


\begin{tabular}{lllll} 
& $\kappa$ & $\theta$ & $\lambda$ & $\rho$ \\
\hline $1 \mathrm{M}$ & 8.23 & $7.96 \%$ & 2.47 & -0.10 \\
$3 \mathrm{M}$ & 5.00 & $6.47 \%$ & 1.32 & -0.19 \\
$6 \mathrm{M}$ & 3.62 & $9.32 \%$ & 1.61 & -0.15 \\
$1 \mathrm{Y}$ & 2.10 & $6.74 \%$ & 1.88 & -0.22 \\
\hline
\end{tabular}

and the calibration and expansion errors are given below (rounded to the nearest basis point):

\begin{tabular}{llllll} 
& 10 Put & 25 Put & ATM & 25 Call & 10 Call \\
\hline $1 \mathrm{M}$ & $6.08[0.08][-0.00]$ & $5.76[0.00][-0.01]$ & $5.53[0.01][0.00]$ & $5.63[-0.07][0.00]$ & $5.81[-0.02][-0.00]$ \\
$3 \mathrm{M}$ & $7.08[0.02][-0.02]$ & $6.53[-0.03][-0.03]$ & $6.15[0.01][0.00]$ & $6.20[-0.03][0.01]$ & $6.44[0.04][-0.01]$ \\
$6 \mathrm{M}$ & $8.19[-0.12][0.06]$ & $7.42[0.02][-0.03]$ & $6.95[0.10][-0.01]$ & $7.00[0.02][0.00]$ & $7.36[-0.05][0.02]$ \\
$1 \mathrm{Y}$ & $9.90[-0.15][0.32]$ & $8.61[0.13][-0.12]$ & $7.95[0.07][-0.07]$ & $8.04[-0.04][-0.02]$ & $8.69[-0.06][0.08]$ \\
\hline
\end{tabular}

Table 4.5: USDJPY Market implied volatility [calibration error] [expansion error] in \%

The median absolute deviation of the calibration error is $4.0 \mathrm{bp}$, and its mean absolute deviation is $5.4 \mathrm{bp}$. The median absolute deviation of the expansion error is $1.5 \mathrm{bp}$, and its mean absolute deviation is $4.1 \mathrm{bp}$. Overall, the median absolute deviation of the total error is $2.0 \mathrm{bp}$, and its mean absolute deviation is $3.9 \mathrm{bp}$.

\subsubsection{Set 3: USD/SGD 04 September $2014\left(S_{0}=1.2541\right)$}

The estimated initial volatility is $V_{0}=3.16 \%$, the estimated piecewise-constant stochastic parameters are given by

\begin{tabular}{lllll} 
& $\kappa$ & $\theta$ & $\lambda$ & $\rho$ \\
\hline $1 \mathrm{M}$ & 2.90 & $4.03 \%$ & 2.30 & 0.49 \\
$2 \mathrm{M}$ & 2.88 & $4.19 \%$ & 1.64 & 0.49 \\
$3 \mathrm{M}$ & 2.85 & $4.44 \%$ & 2.37 & 0.58 \\
$6 \mathrm{M}$ & 2.76 & $4.08 \%$ & 1.68 & 0.51 \\
$1 \mathrm{Y}$ & 2.81 & $4.27 \%$ & 2.31 & 0.67 \\
\hline
\end{tabular}

and the calibration and expansion errors are given below (rounded to the nearest basis point):

\begin{tabular}{llllll} 
& 10 Put & 25 Put & ATM & 25 Call & 10 Call \\
\hline $1 \mathrm{M}$ & $3.06[-0.07][0.05]$ & $3.02[0.06][0.02]$ & $3.30[0.04][0.01]$ & $3.79[0.01][-0.03]$ & $4.43[-0.05][-0.02]$ \\
$2 \mathrm{M}$ & $3.15[-0.02][0.07]$ & $3.15[0.02][0.05]$ & $3.45[0.01][-0.00]$ & $4.10[-0.02][-0.07]$ & $4.84[0.00][-0.03]$ \\
$3 \mathrm{M}$ & $3.23[-0.00][0.07]$ & $3.24[0.00][0.06]$ & $3.57[0.02][-0.00]$ & $4.35[-0.02][-0.09]$ & $5.20[-0.01][0.02]$ \\
$6 \mathrm{M}$ & $3.52[-0.03][0.08]$ & $3.47[-0.02][0.09]$ & $3.80[0.05][0.02]$ & $4.78[0.02][-0.11]$ & $5.85[-0.07][0.17]$ \\
$1 \mathrm{Y}$ & $3.84[-0.05][0.04]$ & $3.78[-0.04][0.11]$ & $4.20[0.12][0.03]$ & $5.40[0.15][-0.07]$ & $6.78[-0.19][0.68]$ \\
\hline
\end{tabular}

Table 4.6: USDSGD Market implied volatility [calibration error] [expansion error] in \%

The median absolute deviation of the calibration error is $2.0 \mathrm{bp}$, and its mean absolute deviation is $4.4 \mathrm{bp}$. 
The median absolute deviation of the expansion error is $5.0 \mathrm{bp}$, and its mean absolute deviation is $8.0 \mathrm{bp}$. Overall, the median absolute deviation of the total error is $7.0 \mathrm{bp}$, and its mean absolute deviation is $7.8 \mathrm{bp}$.

\subsection{Comments}

As shown by Tables 4.4, 4.5 and 4.6, both calibration errors and expansion errors are small overall (only a few basis points of absolute deviation).

The overall calibrations are very good. The worst cases occur for 1 Y maturity and 25 Call strike on Set 1 (+19bp), for 1 Y maturity and 10 Put strike on Set $2(-15 \mathrm{bp})$, and for $1 \mathrm{Y}$ maturity and 10 Call strike on Set $3(-19 \mathrm{bp})$. In other words, they occur for long maturities and strikes far out of the money.

As to the expansion error, it is as expected very small for short maturities and close to the money (ATM), but can get larger for long maturities and far from the money. The worst cases occur for $1 \mathrm{Y}$ maturity and 10 Call strike on Set $1(-63 \mathrm{bp})$, for $1 \mathrm{Y}$ maturity and 10 Put strike on Set $2(+32 \mathrm{bp})$, and again for $1 Y$ maturity and 10 Call strike on Set $3(+68 \mathrm{bp})$. Though these worst case errors can look large in implied volatility terms, the absolute option price values are very small far out of the money, meaning that the actual error is not that large in absolute price terms. Overall, we observe that the expansion error increases with maturity $T$ and volatility of volatility $\lambda$ (as expected from Benhamou et al. [2010]), and with absolute correlation $|\rho|$ (compare Set 2 to the two other sets).

To decrease the calibration errors, one could try more general stochastic volatility models, as discussed in Subsection 2.2, though adding too many parameters may generate overfitting and damage the stability and robustness of the model.

To decrease the expansion error, the most obvious solution is to compute a higher-order expansion (expansion (3.1) is only a second-order expansion). Another idea is to take advantage of the fact that the expansion error is much smaller in practice when the stochastic parameters are constant (only a few bps everywhere). Benhamou et al. [2010] tried to exploit this idea by first calibrating a model with constant parameters for each maturity, and then turning these calibrations into an "equivalent" model with piecewise constant parameters. However, the equivalent piecewise constant model is determined using the same second-order expansion, therefore this two-step calibration procedure does not improve the expansion error of the final model with piecewise-constant parameters.

Depending on the intended application, the accuracy of the present second-order expansion (3.1), as observed in Subsection 4.2, may be sufficient. For example, if one only needs the calibrated stochastic parameters to feed into a more general local-stochastic Inverse Gamma volatility model, then the local volatility component can easily eliminate the residual price discrepancies (cf. Sepp [2014]).

\subsection{Comparison to Heston}

Finally, we compare the Inverse Gamma calibration to a classical Heston stochastic volatility calibration. Subsection 2.3 provided theoretical reasons to favor the Inverse Gamma model over the Heston model, so it is interesting to check if, for example, one of the two models provides significantly better calibrations in practice, as this is one of the important criteria for practical use by the industry. We use the same three datasets, and calibrate the Heston model using the semi-closed-form of Heston [1993].

\subsubsection{Set 1: AUD/USD 17 June $2014\left(S_{0}=0.9335\right)$}

For the Heston model, the estimated initial variance is $V_{0}=0.41 \%$, the estimated piecewise constant stochastic parameters are given by 


\begin{tabular}{cccccc} 
& $\kappa$ & $\theta$ & $\lambda$ & $\rho$ & $2 \kappa \theta / \lambda^{2}$ \\
\hline $1 \mathrm{M}$ & 1.16 & $1.28 \%$ & 0.32 & -0.32 & 0.30 \\
$3 \mathrm{M}$ & 0.97 & $2.32 \%$ & 0.48 & -0.49 & 0.20 \\
$6 \mathrm{M}$ & 1.01 & $1.88 \%$ & 0.51 & -0.54 & 0.15 \\
$1 \mathrm{Y}$ & 1.02 & $1.85 \%$ & 0.51 & -0.52 & 0.14 \\
\hline
\end{tabular}

and the calibration error is given below (rounded to the nearest basis point):

\begin{tabular}{lrllll} 
& \multicolumn{1}{l}{10 Put } & 25 Put & ATM & 25 Call & 10 Call \\
\hline $1 \mathrm{M}$ & $7.48[0.02]$ & $6.87[-0.03]$ & $6.38[0.00]$ & $6.19[-0.04]$ & $6.19[-0.05]$ \\
$3 \mathrm{M}$ & $8.46[0.03]$ & $7.48[-0.07]$ & $6.68[0.01]$ & $6.36[0.03]$ & $6.39[0.07]$ \\
$6 \mathrm{M}$ & $9.93[0.03]$ & $8.43[-0.05]$ & $7.30[0.03]$ & $6.82[0.02]$ & $6.90[-0.06]$ \\
$1 \mathrm{Y}$ & $11.51[-0.04]$ & $9.53[-0.10]$ & $8.05[0.10]$ & $7.47[0.08]$ & $7.57[-0.07]$ \\
\hline
\end{tabular}

Table 4.7: AUDUSD Market implied volatility [Heston calibration error] in \%,

The median absolute deviation of the calibration error is $4.3 \mathrm{bp}$, and its mean absolute deviation is $4.6 \mathrm{bp}$.

\subsubsection{Set 2: USD/JPY 11 June $2014\left(S_{0}=102.00\right)$}

The estimated initial variance is $V_{0}=0.28 \%$, the estimated piecewise constant stochastic parameters are given by

\begin{tabular}{cccccc} 
& $\kappa$ & $\theta$ & $\lambda$ & $\rho$ & $2 \kappa \theta / \lambda^{2}$ \\
\hline $1 \mathrm{M}$ & 1.17 & $1.39 \%$ & 0.23 & -0.11 & 0.62 \\
$3 \mathrm{M}$ & 1.10 & $1.76 \%$ & 0.40 & -0.21 & 0.25 \\
$6 \mathrm{M}$ & 1.09 & $1.73 \%$ & 0.44 & -0.22 & 0.20 \\
$1 \mathrm{Y}$ & 1.04 & $1.92 \%$ & 0.48 & -0.43 & 0.17 \\
\hline
\end{tabular}

and the calibration error is given below (rounded to the nearest basis point):

\begin{tabular}{llllll} 
& 10 Put & 25 Put & ATM & 25 Call & 10 Call \\
\hline $1 \mathrm{M}$ & $6.08[-0.06]$ & $5.76[-0.06]$ & $5.53[0.00]$ & $5.63[-0.10]$ & $5.81[-0.13]$ \\
$3 \mathrm{M}$ & $7.08[-0.00]$ & $6.53[-0.06]$ & $6.15[0.00]$ & $6.20[-0.02]$ & $6.44[0.04]$ \\
$6 \mathrm{M}$ & $8.19[0.13]$ & $7.42[-0.04]$ & $6.95[-0.05]$ & $7.00[-0.05]$ & $7.36[0.06]$ \\
$1 \mathrm{Y}$ & $9.90[0.04]$ & $8.61[-0.03]$ & $7.95[-0.02]$ & $8.04[-0.01]$ & $8.69[0.02]$ \\
\hline
\end{tabular}

Table 4.8: USDJPY Market implied volatility [Heston calibration error] in \%

The median absolute deviation of the calibration error is $4.0 \mathrm{bp}$, and its mean absolute deviation is $4.6 \mathrm{bp}$. 


\subsubsection{Set 3: USD/SGD 04 September $2014\left(S_{0}=1.2541\right)$}

The estimated initial variance is $V_{0}=0.11 \%$, the estimated piecewise constant stochastic parameters are given by

\begin{tabular}{cccccc} 
& $\kappa$ & $\theta$ & $\lambda$ & $\rho$ & $2 \kappa \theta / \lambda^{2}$ \\
\hline $1 \mathrm{M}$ & 1.25 & $0.49 \%$ & 0.28 & 0.41 & 0.16 \\
$2 \mathrm{M}$ & 1.15 & $0.62 \%$ & 0.49 & 0.29 & 0.06 \\
$3 \mathrm{M}$ & 1.24 & $0.54 \%$ & 0.48 & 0.23 & 0.06 \\
$6 \mathrm{M}$ & 1.51 & $0.32 \%$ & 0.52 & 0.44 & 0.04 \\
$1 \mathrm{Y}$ & 1.21 & $0.86 \%$ & 0.49 & -0.26 & 0.09 \\
\hline
\end{tabular}

and the calibration error is given below (rounded to the nearest basis point):

\begin{tabular}{llllll} 
& 10 Put & 25 Put & ATM & 25 Call & 10 Call \\
\hline $1 \mathrm{M}$ & $3.06[-0.07]$ & $3.02[0.02]$ & $3.30[0.00]$ & $3.79[-0.02]$ & $4.43[-0.02]$ \\
$2 \mathrm{M}$ & $3.15[-0.05]$ & $3.15[-0.01]$ & $3.45[-0.01]$ & $4.10[-0.05]$ & $4.84[0.01]$ \\
$3 \mathrm{M}$ & $3.23[0.02]$ & $3.24[-0.00]$ & $3.57[-0.01]$ & $4.35[-0.10]$ & $5.20[-0.00]$ \\
$6 \mathrm{M}$ & $3.52[0.01]$ & $3.47[-0.03]$ & $3.80[0.03]$ & $4.78[-0.04]$ & $5.85[0.13]$ \\
$1 \mathrm{Y}$ & $3.84[0.09]$ & $3.78[-0.04]$ & $4.20[-0.02]$ & $5.40[-0.08]$ & $6.78[0.13]$ \\
\hline
\end{tabular}

Table 4.9: USDSGD Market implied volatility [Heston calibration error] in \%

The median absolute deviation of the calibration error is $2.5 \mathrm{bp}$, and its mean absolute deviation is $4.0 \mathrm{bp}$.

\subsubsection{Result analysis and comparison}

For the three examples, the calibrated parameters do not satisfy the Feller condition: the Feller ratio is much smaller than 1. This is the reason why we could not rely on the Heston closed-form expansion of Benhamou et al. [2010] for calibration. Indeed, when the Feller ratio is so low, the expansion error can be massive. For the USD/JPY parameters, the average absolute expansion error is around 80bp. For the AUD/USD and USD/SGD parameters, the error is so large that some expansion prices can even become negative. When the implied volatility exists, the average absolute expansion error is around 200bp for both AUD/USD and USD/SGD parameters. These large errors were expected, as the expansion approach for the Heston model is not expected to work when the Feller condition is not satisfied (the error analysis in Benhamou et al. [2010] requires the Feller condition to hold). This means that the expansion scheme for the Heston model cannot be used in practice because, as recalled in subsection 2.3, the Feller condition is virtually never satisfied on real-world market data. For this reason, we used the slower semi-closed-form pricing formula of Heston for calibrating the model.

The table below summarizes the calibration error (median and mean, in basis points) for both Inverse Gamma and Heston stochastic volatility models.

\begin{tabular}{lllllll} 
& \multicolumn{2}{l}{ AUD/USD } & \multicolumn{2}{c}{ USD/JPY } & \multicolumn{2}{c}{ USD/SGD } \\
\cline { 2 - 7 } calibration error $(\mathrm{bp})$ & median & mean & median & mean & median & mean \\
\hline Inverse Gamma & 5.0 & 5.7 & 4.0 & 5.4 & 2.0 & 4.4 \\
Heston & 4.3 & 4.6 & 4.0 & 4.6 & 2.5 & 4.0 \\
\hline
\end{tabular}


One can see that the calibration error is of the same order. If anything, the Heston calibration is slightly better $(-0.8 \mathrm{bp}$ on average). Therefore, the calibration error itself does not provide clear and sufficient indication on which stochastic volatility model is a better model in practice. To assess the quality of a stochastic volatility model, the stability of the estimated parameters (the "variance" in statistical terms) is as relevant as the calibration error (the "bias"' in statistical terms). Indeed, a very flexible stochastic volatility model with many parameters is likely to have a very small calibration error on a given market implied volatility curve. However, the parameters of such a model are also likely to be very unstable over time, if recalibrated every day as is common in practice. The stability of parameters is an important criterion for dynamic hedging to work in practice and for the option price to be meaningful at all. This "bias-variance tradeoff" discussion suggests that more numerical tests, in particular stability tests, are needed to further compare the Inverse Gamma and the Heston stochastic volatility models. In particular, a dynamic hedging backtest using the two models for several options over a long time period would be useful. We plan to carry out such tests in the future.

At this stage of the comparison, we recommend the Inverse Gamma model over the Heston model for foreign exchange option pricing for at least three reasons:

- Firstly, even though, based on the three examples studied in this paper, the Heston calibration seems slightly more accurate, the fact that the optimal parameters are very far from satisfying the Feller condition is a major issue, as the very unrealistic volatility distribution resulting from this breach (see Figure 2.1e) is likely to affect the quality of dynamic hedging and the pricing of more exotic options.

- Secondly, from our experience of using the model for more than forty currency pairs, the closedform expansion for Inverse Gamma vanilla options (Theorem 3.1) works quite well on real-world market data (unlike the Heston expansion). If needed, the accuracy of the expansion, already good, can always be enhanced using a higher-order expansion (e.g. 4th order).

- Lastly, the closed-form expansion approach is the fastest method for calibration purposes, and therefore is highly desirable in practice when calibration needs to be performed for hundreds of currency pairs or securities on at least a daily basis.

\section{Conclusion}

This paper has introduced the Inverse Gamma stochastic volatility model, as defined by the volatility dynamics $d V_{t}=\kappa_{t}\left(\theta_{t}-V_{t}\right) d t+\lambda_{t} V_{t} d B_{t}$.

The volatility distribution in this model is more consistent with market dynamics than alternative one factor affine stochastic volatility models such as the Heston model.

We have proposed a closed-form volatility of volatility expansion for the price of a European put option under this stochastic volatility model, and simple and straightforward recursion formulae to instantaneously compute the coefficients of the expansion when the four time-dependent stochastic parameters $\kappa, \theta, \lambda$ and $\rho$ are piecewise constant.

We have demonstrated the viability of the second-order expansion scheme on three test cases from foreign exchange (AUD/USD, USD/JPY and USD/SGD). Both calibration error and expansion error are small overall (only a few basis points of absolute deviation on average).

Potential improvements can be made and have also been discussed in the paper, such as introducing additional stochastic parameters, computing higher order terms in the expansion, performing more comprehensive calibration backtests, and studying the local-stochastic volatility version of this model. We hope to foster further academic research on these non-affine models favoured by industry practitioners, as this paper demonstrates that they not only provide a more accurate representation of market dynamics, but also still be tractable thanks to expansion methods. 


\section{Acknowledgement}

The authors would like to thank Mr Julian Cook of GFI FENICS for many insightful and valuable discussions, and for providing the market data of this research work.

\section{References}

L. Andersen and V. Piterbarg. Moment explosions in stochastic volatility models. Finance and Stochastics, 11(1):29-50, 2007. (Cited on page 8)

G. Barone-Adesi, H. Rasmussen, and C. Ravanelli. An option pricing formula for the GARCH diffusion model. Computational Statistics and Data Analysis, 49(2):287-310, 2005. (Cited on pages 22 and 23)

E. Benhamou, E. Gobet, and M. Miri. Time dependent Heston model. SIAM Journal on Financial Mathematics, 1(1):289-325, 2010. (Cited on pages 8, 9, 10, 16, 18, 25, 27, 28, and 30)

J.-P. Bouchaud and M. Potters. Theory of Financial Risk and Derivative Pricing: From Statistical Physics to Risk Management. Cambridge University Press, 2003. (Cited on page 7)

P. Christoffersen, K. Jacobs, and K. Mimouni. Volatility dynamics for the S\&P500: Evidence from realized volatility, daily returns, and option prices. Review of Financial Studies, 23(8):3141-3189, 2010. (Cited on page 7)

I. Clark. Foreign exchange option pricing: A practitioners's guide. Wiley Finance. John Wiley \& Sons, 2011. (Cited on page 5)

J. da Fonseca and M. Grasselli. Riding on the smiles. Quantitative Finance, 11(11):1609-1632, 2011. (Cited on page 5)

F. Fornari and A. Mele. Recovering the probability density function of asset prices using GARCH as diffusion approximations. Journal of Empirical Finance, 8(1):83-110, 2001. (Cited on pages 3 and 7)

F. Fornari and A. Mele. Approximating volatility diffusions with CEV-ARCH models. Journal of Economic Dynamics and Control, 30(6):931-966, 2006. (Cited on page 7)

M. Gander and D. Stephens. Stochastic volatility modelling in continuous time with general marginal distributions: Inference, prediction and model selection. Journal of Statistical Planning and Inference, 137(10):3068-3081, 2007. (Cited on page 7)

J. Gatheral. Further developments in volatility derivatives pricing. In Global Derivatives, 2007. (Cited on page 3 )

J. Gatheral. Consistent modeling of SPX and VIX options. In Bachelier Congress, 2008. (Cited on pages 3 and 7 )

A. Hansis. Affine versus non-affine stochastic volatility and the impact on asset allocation. SSRN:1545783, 2010. (Cited on page 8)

P. Henry-Labordère. Analysis, Geometry, and Modeling in Finance: Advanced Methods in Option Pricing. Financial Mathematics Series. Chapman \& Hall/CRC, 2008. (Cited on page 4)

P. Henry-Labordère. Calibration of local stochastic volatility models to market smiles. Risk, 22(9): 112-117, 2009. (Cited on page 3)

S. Heston. A closed-form solution for options with stochastic volatility with applications to bond and currency options. Review of Financial Studies, 6(2):327-343, 1993. (Cited on pages 5 and 16) 
A. Itkin. New solvable stochastic volatility models for pricing volatility derivatives. Review of Derivatives Research, 16(2):111-134, 2013. (Cited on page 4)

Y. Jerbi. Methodology for stochastic volatility process calibration application to the CAC 40 index. Journal of Statistical Computation and Simulation, 83(3):417-433, 2011. (Cited on page 4)

A. Kaeck and C. Alexander. Volatility dynamics for the S\&P 500: Further evidence from non-affine, multi-factor jump diffusions. Journal of Banking \& Finance, 36(11):3110-3121, 2012. (Cited on page 7)

C. Kahl and P. Jackel. Fast strong approximation Monte Carlo schemes for stochastic volatility models. Quantitative Finance, 6(6):513-536, 2006. (Cited on page 12)

A. Lewis. Option valuation under stochastic volatility with Mathematica code. Finance Press, 2000. (Cited on page 5)

C. Lo, H. Lee, and C. Hui. A simple approach for pricing barrier options with time-dependent parameters. Quantitative Finance, 3(2):98-107, 2003. (Cited on page 9)

T. Ma and R. Serota. A model for stock returns and volatility. Physica A: Statistical Mechanics and its Applications, 398:89-115, 2014. (Cited on pages 4 and 7)

F. Rapisarda. Pricing barriers on underlyings with time-dependent parameters. Technical report, Banca IMI, 2003. (Cited on page 9)

A. Ribeiro and R. Poulsen. Approximation behoves calibration. Quantitative Finance Letters, 1(1): 36-40, 2013. (Cited on page 5)

J. Rojo. On tail categorization of probability laws. Journal of the American Statistical Association, 91 (433):378-384, 1996. (Cited on page 5)

R. Schöbel and J. Zhu. Stochastic volatility with an Ornstein-Uhlenbeck process: an extension. European Finance Review, 3(1):23-46, 1999. (Cited on page 5)

A. Sepp. Empirical calibration and minimum-variance delta under log-normal stochastic volatility dynamics. SSRN:2387845, 2014. (Cited on pages 4, 8, 16, and 23)

A. Sepp. Log-normal stochastic volatility model: Pricing of vanilla options and econometric estimation. SSRN:2522425, 2015. (Cited on pages 4, 8, and 23)

K. Shiraya and A. Takahashi. Pricing average options on commodities. Journal of Futures Markets, 31 (5):407-439, 2011. (Cited on page 4)

K. Shiraya and A. Takahashi. Pricing multiasset cross-currency options. Journal of Futures Markets, 34(1):1-19, 2014. (Cited on page 4)

G. Tataru and T. Fisher. The Bloomberg stochastic local volatility model for FX exotics. Technical report, Bloomberg, 2012. (Cited on page 8)

J. Wiggins. Option values under stochastic volatility: Theory and empirical estimates. Journal of Financial Economics, 19(2):351-372, 1987. (Cited on page 5)

B. Zhao. Inhomogeneous Geometric Brownian Motions. SSRN:1429449, 2009. (Cited on pages 12 and 23) 


\section{A Stationary distribution of volatility}

\section{A.1 Heston}

For the Heston stochastic volatility model with constant coefficients,

$$
\begin{aligned}
d S_{t} & =\left(r_{d}-r_{f}\right) S_{t} d t+\sqrt{V_{t}} S_{t} d W_{t} \\
d V_{t} & =\kappa\left(\theta-V_{t}\right) d t+\lambda \sqrt{V_{t}} d B_{t} \\
d\langle W, B\rangle_{t} & =\rho d t,
\end{aligned}
$$

the long-run distribution of the variance $V_{t}$ is a Gamma distribution with parameters $k_{\Gamma}=\beta \theta$ and $\theta_{\Gamma}=\frac{1}{\beta}$, where $\beta:=\frac{2 \kappa}{\lambda^{2}}$. Remark that the probability density function of $V_{t}$ can reach 0 if $\beta \theta<1$ (Feller condition).

Consequently, the long-run Heston volatility $Y_{t}=\sqrt{V_{t}}$ has a generalized Chi distribution

$$
p_{\chi}(x ; a, b, \nu)=\frac{1}{2^{\frac{\nu}{2}-1} b \Gamma\left(\frac{\nu}{2}\right)}\left(\frac{x-a}{b}\right)^{\nu-1} e^{-\frac{1}{2}\left(\frac{x-a}{b}\right)^{2}}
$$

with parameters $a=0, b=\frac{1}{\sqrt{2 \beta}}$ and $\nu=2 \beta \theta$.

In particular, the moments of the long-run volatility $Y_{t}=\sqrt{V_{t}}$ are given by:

$$
\begin{aligned}
\mathbb{E}\left[Y_{t}\right] & \rightarrow \frac{\Gamma\left(k_{\Gamma}+\frac{1}{2}\right)}{\Gamma\left(k_{\Gamma}\right)} \sqrt{\theta_{\Gamma}}=\frac{\Gamma\left(\beta \theta+\frac{1}{2}\right)}{\Gamma(\beta \theta) \sqrt{\beta \theta}} \sqrt{\theta} \\
\mathbb{E}\left[Y_{t}^{2}\right] & \rightarrow k_{\Gamma} \theta_{\Gamma}=\theta
\end{aligned}
$$

\section{A.2 Inverse Gamma}

For the Inverse Gamma stochastic volatility model with constant coefficients,

$$
\begin{aligned}
d S_{t} & =\left(r_{d}-r_{f}\right) S_{t} d t+V_{t} S_{t} d W_{t} \\
d V_{t} & =\kappa\left(\theta-V_{t}\right) d t+\lambda V_{t} d B_{t} \\
d\langle W, B\rangle_{t} & =\rho d t,
\end{aligned}
$$

the long-run distribution of the volatility process $V_{t}$ is an inverse Gamma distribution

$$
p_{\Gamma^{-1}}(x ; \alpha, \beta)=\frac{\beta^{\alpha}}{\Gamma(\alpha)} x^{-\alpha-1} e^{-\frac{\beta}{x}}
$$

with parameters $\alpha_{\Gamma^{-1}}=1+\beta$ and $\beta_{\Gamma^{-1}}=\beta \theta$, where $\beta:=\frac{2 \kappa}{\lambda^{2}}$ (see Barone-Adesi et al. [2005]). Therefore

$$
\begin{aligned}
\mathbb{E}\left[V_{t}\right] & \rightarrow \frac{\beta_{\Gamma^{-1}}}{\alpha_{\Gamma^{-1}}-1}=\theta(\text { iff } \beta>0) \\
\operatorname{Var}\left[V_{t}\right] & \rightarrow \frac{\beta_{\Gamma^{-1}}^{2}}{\left(\alpha_{\Gamma^{-1}}-1\right)^{2}\left(\alpha_{\Gamma^{-1}}-2\right)}=\frac{\theta^{2}}{\beta-1}(\text { iff } \beta>1)
\end{aligned}
$$

\section{B The Log-Normal terminology}

There exists three different volatility dynamics that have been called log-normal in the literature:

$$
\begin{aligned}
d V_{t} & =\kappa V_{t} d t+\lambda V_{t} d B_{t} \\
d V_{t} & =\kappa\left(\theta-V_{t}\right) d t+\lambda V_{t} d B_{t} \\
d \log \left(V_{t}\right) & =\kappa\left(\theta-\log \left(V_{t}\right)\right) d t+\lambda d B_{t}
\end{aligned}
$$


In the first formulation (B.1), the volatility is modeled by a geometric Brownian motion, which is $\log$-normally distributed. It is a special case of SABR model (with $\beta=1$ ). It is not mean-reverting.

In the last formulation (B.3), the logarithm of the volatility is an Ornstein-Uhlenbeck process, which has a normally-distributed stationary distribution. Therefore the long-term distribution of the volatility $V_{t}$ is indeed log-normally distributed.

The intermediate formulation (B.2) combines characteristics from a geometric Brownian motion (the volatility of volatility $\lambda V_{t}$ is proportional to the volatility $V_{t}$ ) and from an exponential OrnsteinUhlenbeck (the mean-reversion effect towards a level $\theta$ ). However the stationary distribution of the volatility is not a log-normal distribution but an inverse Gamma distribution (Barone-Adesi et al. [2005], Zhao [2009], Sepp [2014, 2015]).

Practitioners sometimes refer to (B.2) as a "Log-Normal" or "mean-reverting Log-Normal" stochastic volatility model, but as this terminology can be ambiguous and misleading, we choose to call it Inverse Gamma stochastic volatility model, which is consistent with the stationary distribution of the volatility (B.2).

\section{Proof of main expansion}

In the IGa model (2.1), factoring out the drift rates $r_{d}$ and $r_{f}$, the dynamics of the log-spot $X_{t}$ reads

$$
\begin{aligned}
d X_{t} & =-\frac{V_{t}^{2}}{2} d t+V_{t} d W_{t}, \quad X_{0}=x_{0} \\
d V_{t} & =\kappa_{t}\left(\theta_{t}-V_{t}\right) d t+\lambda_{t} V_{t} d B_{t}, \quad V_{0}=v_{0} \\
d\langle W, B\rangle_{t} & =\rho_{t} d t,
\end{aligned}
$$

where $x_{0}=\log \left(S_{0}\right)$. Define a perturbed process $\left(X^{\varepsilon}, V^{\varepsilon}\right)$ as follows

$$
\begin{aligned}
d X_{t}^{\varepsilon} & =-\frac{\left(V_{t}^{\varepsilon}\right)^{2}}{2} d t+V_{t}^{\varepsilon} d W_{t}, \quad X_{0}^{\varepsilon}=x_{0} \\
d V_{t}^{\varepsilon} & =\kappa_{t}\left(\theta_{t}-V_{t}^{\varepsilon}\right) d t+\varepsilon \lambda_{t} V_{t}^{\varepsilon} d B_{t}, \quad V_{0}^{\varepsilon}=v_{0} \\
d\langle W, B\rangle_{t} & =\rho_{t} d t
\end{aligned}
$$

and define

$$
g(\varepsilon)=\exp \left(-\int_{0}^{T} r_{d}(t) d t\right) \mathbb{E}\left[\left(K-\exp \left(-\int_{0}^{T}\left(r_{d}(t)-r_{f}(t)\right) d t\right)+X_{T}^{\varepsilon}\right)_{+}\right],
$$

so that $g(1)=P_{I G a}$, the price of European put with IGa stochastic volatility, which is the quantity we want to compute. Remark that $g(0)$ reduces to a Black-Scholes price.

The expression (C.3) for $g(\varepsilon)$ can be simplified. Remark that $W_{t}$ can be decomposed into

$$
W_{t}=\rho_{t} B_{t}+\sqrt{1-\rho_{t}^{2}} d B_{t}^{\perp}
$$

where $B^{\perp}$ is a Brownian motion independent from $B$. Therefore

$$
X_{t}^{\varepsilon}=x_{0}+\int_{0}^{T} \rho_{t} V_{t}^{\varepsilon} d B_{t}-\frac{1}{2} \int_{0}^{T}\left(V_{t}^{\varepsilon}\right)^{2} d t+\int_{0}^{T} \sqrt{1-\rho_{t}^{2}} V_{t}^{\varepsilon} d B_{t}^{\perp} .
$$

Let $\mathcal{F}^{B}=\left(\mathcal{F}_{t}^{B}\right)_{0 \leq t \leq T}$ be the filtration generated by $B$. One can see that $X_{T}^{\varepsilon} \mid \mathcal{F}_{T}^{B}$ has a Gaussian distribution with mean $x_{0}+\int_{0}^{T} \rho_{t} V_{t}^{\varepsilon} d B_{t}-\frac{1}{2} \int_{0}^{T}\left(V_{t}^{\varepsilon}\right)^{2} d t=\left\{x_{0}+\int_{0}^{T} \rho_{t} V_{t}^{\varepsilon} d B_{t}-\frac{1}{2} \int_{0}^{T}\left(\rho_{t} V_{t}^{\varepsilon}\right)^{2} d t\right\}-\frac{1}{2} \int_{0}^{T}(1-$ 
$\left.\rho_{t}^{2}\right)\left(V_{t}^{\varepsilon}\right)^{2} d t$ and variance $\int_{0}^{T}\left(1-\rho_{t}^{2}\right)\left(V_{t}^{\varepsilon}\right)^{2} d t$. Consequently,

$$
\begin{aligned}
g(\varepsilon) & =\exp \left(-\int_{0}^{T} r_{d}(t) d t\right) \mathbb{E}\left[\mathbb{E}\left[\left(K-\exp \left(-\int_{0}^{T}\left(r_{d}(t)-r_{f}(t)\right) d t\right)+X_{T}^{\varepsilon}\right) \mid \mathcal{F}_{T}^{B}\right]\right] \\
& =\mathbb{E}\left[P_{B S}\left(x_{0}+\int_{0}^{T} \rho_{t} V_{t}^{\varepsilon} d B_{t}-\frac{1}{2} \int_{0}^{T}\left(\rho_{t} V_{t}^{\varepsilon}\right)^{2} d t, \int_{0}^{T}\left(1-\rho_{t}^{2}\right)\left(V_{t}^{\varepsilon}\right)^{2} d t\right)\right]
\end{aligned}
$$

where $P_{B S}(x, y)$ is the Black-Scholes price of a European put option with spot $e^{x}$ and integrated variance $y$ (equation (3.6)).

For any non-negative integer $n$, define $V_{n, t}^{\varepsilon}:=\frac{\partial^{n} V_{t}^{\varepsilon}}{\partial \varepsilon^{n}}$ and $V_{n, t}:=\left.\frac{\partial^{n} V_{t}^{\varepsilon}}{\partial \varepsilon^{n}}\right|_{\varepsilon=0}$. One can check that

$$
\begin{aligned}
& d V_{0, t}^{\varepsilon}=\kappa_{t}\left(\theta_{t}-V_{0, t}^{\varepsilon}\right) d t+\varepsilon \lambda_{t} V_{0, t}^{\varepsilon} d B_{t}, \quad V_{0,0}^{\varepsilon}=v_{0} \\
& d V_{n, t}^{\varepsilon}=-\kappa_{t} V_{n, t}^{\varepsilon} d t+n \lambda_{t} V_{n-1, t}^{\varepsilon} d B_{t}+\varepsilon \lambda_{t} V_{n, t}^{\varepsilon} d B_{t}, \quad V_{n, 0}^{\varepsilon}=0, \quad n \geq 1
\end{aligned}
$$

and

$$
\begin{aligned}
v_{0, t} & =e^{-\int_{0}^{t} \kappa_{z} d z}\left(v_{0}+\int_{0}^{t} \kappa_{s} \theta_{s} e^{\int_{0}^{s} \kappa_{z} d z} d s\right) \\
V_{n, t} & =e^{-\int_{0}^{t} \kappa_{z} d z} \int_{0}^{t} e^{\int_{0}^{s} \kappa_{z} d z} n \lambda_{s} V_{n-1, s} d B_{s}, \quad n \geq 1
\end{aligned}
$$

where $v_{0, t}:=V_{0, t}$ is deterministic.

At this point, the main idea of the proof is to approximate $g(1)$ using a Taylor expansion of $g(\varepsilon)$ around $\varepsilon=0$, as $g$ and its derivatives reduce to a Black-Scholes formula when $\varepsilon=0$.

Thus, we approximate $V_{t}=V_{t}^{1}(\varepsilon=1)$ using $V_{t}^{0}=v_{0, t}(\varepsilon=0)$ by applying the Taylor formula to the function $\varepsilon \mapsto V_{t}^{\varepsilon}$

$$
V_{t}=v_{0, t}+V_{1, t}+\frac{1}{2} V_{2, t}+\ldots
$$

Doing the same to the function $\varepsilon \mapsto\left(V_{t}^{\varepsilon}\right)^{2}$ yields

$$
\left(V_{t}\right)^{2}=\left(v_{0, t}\right)^{2}+2 v_{0, t} V_{1, t}+\left(v_{0, t} V_{2, t}+\left(V_{1, t}\right)^{2}\right)+\ldots
$$

To simplify notations, define, for $i \geq 0$ and $j \geq 0$,

$$
\frac{\partial^{i+j} \tilde{P}_{B S}}{\partial x^{i} y^{j}}:=\frac{\partial^{i+j} P_{B S}}{\partial x^{i} y^{j}}\left(x_{0}+\int_{0}^{T} \rho_{t} v_{0, t} d B_{t}-\frac{1}{2} \int_{0}^{T} \rho_{t}^{2} v_{0, t}^{2} d t, \int_{0}^{T}\left(1-\rho_{t}^{2}\right) v_{0, t}^{2} d t\right) .
$$

Then, the second-order Taylor expansion of $g(\varepsilon)$ around $\varepsilon=0$, valued at $\varepsilon=1$, reads (keeping only 
second-order terms)

$$
\begin{aligned}
g(1) & =\mathbb{E}\left[\tilde{P}_{B S}\right] \\
\left(C_{x}:=\right) & +\mathbb{E}\left[\frac{\partial \tilde{P}_{B S}}{\partial x}\left\{\int_{0}^{T} \rho_{t}\left(V_{1, t}+\frac{1}{2} V_{2, t}\right) d B_{t}-\frac{1}{2} \int_{0}^{T} \rho_{t}^{2}\left(2 v_{0, t} V_{1, t}+V_{1, t}^{2}+v_{0, t} V_{2, t}\right) d t\right\}\right] \\
\left(C_{y}:=\right) & +\mathbb{E}\left[\frac{\partial \tilde{P}_{B S}}{\partial y}\left\{\int_{0}^{T}\left(1-\rho_{t}^{2}\right)\left(2 v_{0, t} V_{1, t}+V_{1, t}^{2}+v_{0, t} V_{2, t}\right) d t\right\}\right] \\
\left(C_{x x}:=\right) & \left.+\frac{1}{2} \mathbb{E}\left[\frac{\partial^{2} \tilde{P}_{B S}}{\partial x^{2}}\left\{\int_{0}^{T} \rho_{t}\left(V_{1, t}\right) d B_{t}-\frac{1}{2} \int_{0}^{T} \rho_{t}^{2}\left(2 v_{0, t} V_{1, t}\right) d t\right\}\right\}^{2}\right] \\
\left(C_{y y}:=\right) & +\frac{1}{2} \mathbb{E}\left[\frac{\partial^{2} \tilde{P}_{B S}}{\partial y^{2}}\left\{\int_{0}^{T}\left(1-\rho_{t}^{2}\right)\left(2 v_{0, t} V_{1, t}\right) d t\right\}^{2}\right] \\
\left(C_{x y}:=\right) & +\mathbb{E}\left[\frac{\partial^{2} \tilde{P}_{B S}}{\partial x \partial y}\left\{\int_{0}^{T} \rho_{t}\left(V_{1, t}\right) d B_{t}-\frac{1}{2} \int_{0}^{T} \rho_{t}^{2}\left(2 v_{0, t} V_{1, t}\right) d t\right\}\left\{\int_{0}^{T}\left(1-\rho_{t}^{2}\right)\left(2 v_{0, t} V_{1, t}\right) d t\right\}\right] \\
& +\mathcal{E},
\end{aligned}
$$

where $\mathcal{E}$ is the expansion error. From definition (C.7), taking $\varepsilon=0$ in (C.4) shows that

$$
\mathbb{E}\left[\tilde{P}_{B S}\right]=P_{B S}\left(x_{0}, \int_{0}^{T} v_{0, t}^{2} d t\right) .
$$

Similarly,

$$
\mathbb{E}\left[\frac{\partial^{i+j} \tilde{P}_{B S}}{\partial x^{i} y^{j}}\right]=\frac{\partial^{i+j} P_{B S}}{\partial x^{i} y^{j}}\left(x_{0}, \int_{0}^{T} v_{0, t}^{2} d t\right) .
$$

The next part of this appendix is devoted to the computation of all the constants $C_{x}, C_{y}, C_{x x}, C_{y y}$ and $C_{x y}$. Our main tools will be the following relation between Black-Sholes greeks:

$$
\frac{\partial P_{B S}}{\partial y}(x, y)=\frac{1}{2}\left(\frac{\partial^{2} P_{B S}}{\partial x^{2}}(x, y)-\frac{\partial P_{B S}}{\partial x}(x, y)\right),
$$

the following product identity between two stochastic processes $X$ and $Y$ :

$$
X_{T} Y_{T}=X_{0} Y_{0}+\int_{0}^{T}\left(X_{t} d Y_{t}+Y_{t} d X_{t}+d\langle X, Y\rangle_{t}\right)
$$

and the following Lemma:

Lemma C.1. If $G=l\left(\int_{0}^{T} \rho_{u} v_{0, u} d B_{u}\right)$ for a differentiable function $l$ with derivative $l^{(1)}$, then its first Malliavin derivative $D^{B}(G)=\left(D_{s}^{B}(G)\right)_{s \geq 0}$ is equal to $D_{s}^{B}(G)=l^{(1)}\left(\int_{0}^{T} \rho_{u} v_{0, u} d B_{u}\right) \rho_{s} v_{0, s} \mathbb{1}\{s \leq T\}$. Therefore, using Lemma 5.2 from Benhamou et al. [2010],

$$
\mathbb{E}\left[l\left(\int_{0}^{T} \rho_{u} v_{0, u} d B_{u}\right)\left(\int_{0}^{t} \alpha_{s} d B_{s}\right)\right]=\mathbb{E}\left[l^{(1)}\left(\int_{0}^{T} \rho_{u} v_{0, u} d B_{u}\right)\left(\int_{0}^{t} \rho_{s} v_{0, s} \alpha_{s} d s\right)\right]
$$

for $0 \leq t \leq T$. Most of the time, we will use (C.13) with $t=T$.

- Computation of $C_{x}$ : 
Using (C.13),

$$
\mathbb{E}\left[\frac{\partial \tilde{P}_{B S}}{\partial x}\left\{\int_{0}^{T} \rho_{t}\left(V_{1, t}+\frac{1}{2} V_{2, t}\right) d B_{t}\right\}\right]=\mathbb{E}\left[\frac{\partial^{2} \tilde{P}_{B S}}{\partial x^{2}}\left\{\int_{0}^{T} \rho_{t}^{2}\left(v_{0, t} V_{1, t}+\frac{1}{2} v_{0, t} V_{2, t}\right) d t\right\}\right] .
$$

Therefore, using (C.11),

$$
C_{x}=\mathbb{E}\left[\frac{\partial \tilde{P}_{B S}}{\partial y}\left\{\int_{0}^{T} \rho_{t}^{2}\left(2 v_{0, t} V_{1, t}+v_{0, t} V_{2, t}\right) d t\right\}\right]-\mathbb{E}\left[\frac{\partial \tilde{P}_{B S}}{\partial x}\left\{\frac{1}{2} \int_{0}^{T} \rho_{t}^{2} V_{1, t}^{2} d t\right\}\right]
$$

- Computation of $C_{x x}$ :

Using (C.12), then (C.13), and finally (C.11),

$$
\begin{aligned}
C_{x x} & =\mathbb{E}\left[\frac{\partial^{2} \tilde{P}_{B S}}{\partial x^{2}} \int_{0}^{T}\left\{\int_{0}^{t} \rho_{s} V_{1, s} d B_{s}-\int_{0}^{t} \rho_{s}^{2} v_{0, s} V_{1, s} d s\right\}\left\{\rho_{t} V_{1, t} d B_{t}-\rho_{t}^{2} v_{0, t} V_{1, t} d t\right\}\right]+\frac{1}{2} \mathbb{E}\left[\frac{\partial^{2} \tilde{P}_{B S}}{\partial x^{2}} \int_{0}^{T} \rho_{t}^{2} V_{1, t}^{2} d t\right] \\
& =\mathbb{E}\left[\left(\frac{\partial^{3} \tilde{P}_{B S}}{\partial x^{3}}-\frac{\partial^{2} \tilde{P}_{B S}}{\partial x^{2}}\right) \int_{0}^{T}\left\{\int_{0}^{t} \rho_{s} V_{1, s} d B_{s}-\int_{0}^{t} \rho_{s}^{2} v_{0, s} V_{1, s} d s\right\} \rho_{t}^{2} v_{0, t} V_{1, t} d t\right]+\frac{1}{2} \mathbb{E}\left[\frac{\partial^{2} \tilde{P}_{B S}}{\partial x^{2}} \int_{0}^{T} \rho_{t}^{2} V_{1, t}^{2} d t\right] \\
& =2 \mathbb{E}\left[\frac{\partial^{2} \tilde{P}_{B S}}{\partial x \partial y} \int_{0}^{T}\left\{\int_{0}^{t} \rho_{s} V_{1, s} d B_{s}-\int_{0}^{t} \rho_{s}^{2} v_{0, s} V_{1, s} d s\right\} \rho_{t}^{2} v_{0, t} V_{1, t} d t\right]+\frac{1}{2} \mathbb{E}\left[\frac{\partial^{2} \tilde{P}_{B S}}{\partial x^{2}} \int_{0}^{T} \rho_{t}^{2} V_{1, t}^{2} d t\right]
\end{aligned}
$$

Therefore, so far, using (C.11),

$$
\begin{aligned}
C_{x}+C_{y}+C_{x x} & =\mathbb{E}\left[\frac{\partial \tilde{P}_{B S}}{\partial y}\left\{\int_{0}^{T}\left(2 v_{0, t} V_{1, t}+V_{1, t}^{2}+v_{0, t} V_{2, t}\right) d t\right\}\right] \\
\left(\tilde{C}_{x y}:=\right) & +2 \mathbb{E}\left[\frac{\partial^{2} \tilde{P}_{B S}}{\partial x \partial y} \int_{0}^{T}\left\{\int_{0}^{t} \rho_{s} V_{1, s} d B_{s}-\int_{0}^{t} \rho_{s}^{2} v_{0, s} V_{1, s} d s\right\} \rho_{t}^{2} v_{0, t} V_{1, t} d t\right]
\end{aligned}
$$

- Computation of $C_{x y}$ :

Using (C.12),

$$
\begin{aligned}
C_{x y} & =\mathbb{E}\left[\frac{\partial^{2} \tilde{P}_{B S}}{\partial x \partial y}\left\{\int_{0}^{T} \rho_{t} V_{1, t} d B_{t}-\int_{0}^{T} \rho_{t}^{2} v_{0, t} V_{1, t} d t\right\}\left\{\int_{0}^{T}\left(1-\rho_{t}^{2}\right)\left(2 v_{0, t} V_{1, t}\right) d t\right\}\right] \\
& =\mathbb{E}\left[\frac{\partial^{2} \tilde{P}_{B S}}{\partial x \partial y} \int_{0}^{T}\left(\int_{0}^{t} \rho_{s} V_{1, s} d B_{s}-\int_{0}^{t} \rho_{s}^{2} v_{0, s} V_{1, s} d s\right)\left(1-\rho_{t}^{2}\right)\left(2 v_{0, t} V_{1, t}\right) d t\right] \\
& +\mathbb{E}\left[\frac{\partial^{2} \tilde{P}_{B S}}{\partial x \partial y} \int_{0}^{T}\left\{\int_{0}^{t}\left(1-\rho_{s}^{2}\right)\left(2 v_{0, s} V_{1, s}\right) d s\right\}\left\{\rho_{t} V_{1, t} d B_{t}-\rho_{t}^{2} v_{0, t} V_{1, t} d t\right\}\right]
\end{aligned}
$$

Using (C.13),

$$
\begin{aligned}
C_{x y}+\tilde{C}_{x y} & =\mathbb{E}\left[\frac{\partial^{2} \tilde{P}_{B S}}{\partial x \partial y} \int_{0}^{T}\left(\int_{0}^{t} \rho_{s} V_{1, s} d B_{s}-\int_{0}^{t} \rho_{s}^{2} v_{0, s} V_{1, s} d s\right)\left(2 v_{0, t} V_{1, t}\right) d t\right] \\
& +\mathbb{E}\left[\frac{\partial^{2} \tilde{P}_{B S}}{\partial x \partial y} \int_{0}^{T}\left\{\int_{0}^{t}\left(1-\rho_{s}^{2}\right)\left(2 v_{0, s} V_{1, s}\right) d s\right\}\left\{\rho_{t} V_{1, t} d B_{t}-\rho_{t}^{2} v_{0, t} V_{1, t} d t\right\}\right]
\end{aligned}
$$


Define $G=\frac{\partial^{2} \tilde{P}_{B S}}{\partial x \partial y} v_{0, t} V_{1, t}$. Then $D_{s}^{B}(G)$, its first Malliavin derivative w.r.t. $B$, is given by

$$
\begin{aligned}
D_{s}^{B}(G) & =v_{0, t} V_{1, t} D_{s}^{B}\left(\frac{\partial^{2} \tilde{P}_{B S}}{\partial x \partial y}\right)+\frac{\partial^{2} \tilde{P}_{B S}}{\partial x \partial y} v_{0, t} D_{s}^{B}\left(V_{1, t}\right) \\
& =v_{0, t} V_{1, t} \frac{\partial^{3} \tilde{P}_{B S}}{\partial x^{2} \partial y} \rho_{s} v_{0, s} \mathbb{1}\{s \leq T\}+\frac{\partial^{2} \tilde{P}_{B S}}{\partial x \partial y} v_{0, t} e^{-\int_{0}^{t} \kappa_{z} d z} e^{\int_{0}^{s} \kappa_{z} d z} \lambda_{s} v_{0, s} \mathbb{1}\{s \leq t\}
\end{aligned}
$$

using the definition of $V_{1, t}$ (C.6). Therefore, using Lemma 5.2 in Benhamou et al. [2010],

$$
\begin{aligned}
& 2 \int_{0}^{T} \mathbb{E}\left[\frac{\partial^{2} \tilde{P}_{B S}}{\partial x \partial y} v_{0, t} V_{1, t}\left(\int_{0}^{t} \rho_{s} V_{1, s} d B_{s}\right)\right] d t \\
= & 2 \int_{0}^{T} \mathbb{E}\left[G\left\{\int_{0}^{t} \rho_{s} V_{1, s} d B_{s}\right\}\right] d t \\
= & 2 \int_{0}^{T} \mathbb{E}\left[\int_{0}^{t} \rho_{s} V_{1, s} D_{s}^{B}(G) d s\right] d t \\
= & 2 \mathbb{E}\left[\frac{\partial^{3} \tilde{P}_{B S}}{\partial x^{2} \partial y} \int_{0}^{T}\left(\int_{0}^{t} \rho_{s}^{2} v_{0, s} V_{1, s} d s\right) v_{0, t} V_{1, t} d t\right]+2 \int_{0}^{T} v_{0, t} e^{-\int_{0}^{t} \kappa_{z} d z} \mathbb{E}\left[\frac{\partial^{2} \tilde{P}_{B S}}{\partial x \partial y} \int_{0}^{t} e^{\int_{0}^{s} \kappa_{z} d z} \lambda_{s} \rho_{s} v_{0, s} V_{1, s} d s\right] d t \\
= & 2 \mathbb{E}\left[\frac{\partial^{3} \tilde{P}_{B S}}{\partial x^{2} \partial y} \int_{0}^{T}\left(\int_{0}^{t} \rho_{s}^{2} v_{0, s} V_{1, s} d s\right) v_{0, t} V_{1, t} d t\right]+\mathbb{E}\left[\frac{\partial \tilde{P}_{B S}}{\partial y} \int_{0}^{T} v_{0, t} V_{2, t} d t\right]
\end{aligned}
$$

where we used, for the last equality, equation (C.13) and the definition of $V_{2, t}$ (equation (C.6)). Therefore, using (C.13) and then (C.11),

$$
\begin{aligned}
C_{x y}+\tilde{C}_{x y} & =4 \mathbb{E}\left[\frac{\partial^{2} \tilde{P}_{B S}}{\partial y^{2}} \int_{0}^{T}\left(\int_{0}^{t} \rho_{s}^{2} v_{0, s} V_{1, s} d s\right) v_{0, t} V_{1, t} d t\right] \\
& +4 \mathbb{E}\left[\frac{\partial^{2} \tilde{P}_{B S}}{\partial y^{2}} \int_{0}^{T}\left\{\int_{0}^{t}\left(1-\rho_{s}^{2}\right) v_{0, s} V_{1, s} d s\right\} \rho_{t}^{2} v_{0, t} V_{1, t} d t\right] \\
& +\mathbb{E}\left[\frac{\partial \tilde{P}_{B S}}{\partial y} \int_{0}^{T} v_{0, t} V_{2, t} d t\right]
\end{aligned}
$$

Using (C.12),

$$
C_{y y}=4 \mathbb{E}\left[\frac{\partial^{2} \tilde{P}_{B S}}{\partial y^{2}} \int_{0}^{T}\left\{\int_{0}^{t}\left(1-\rho_{s}^{2}\right) v_{0, s} V_{1, s} d s\right\}\left(1-\rho_{t}^{2}\right) v_{0, t} V_{1, t} d t\right] .
$$

Thus

$$
\begin{aligned}
C_{x y}+\tilde{C}_{x y}+C_{y y} & =4 \mathbb{E}\left[\frac{\partial^{2} \tilde{P}_{B S}}{\partial y^{2}} \int_{0}^{T}\left(\int_{0}^{t} v_{0, s} V_{1, s} d s\right) v_{0, t} V_{1, t} d t\right]+\mathbb{E}\left[\frac{\partial \tilde{P}_{B S}}{\partial y} \int_{0}^{T} v_{0, t} V_{2, t} d t\right] \\
& =2 \mathbb{E}\left[\frac{\partial^{2} \tilde{P}_{B S}}{\partial y^{2}}\left\{\int_{0}^{T} v_{0, t} V_{1, t} d t\right\}^{2}\right]+\mathbb{E}\left[\frac{\partial \tilde{P}_{B S}}{\partial y} \int_{0}^{T} v_{0, t} V_{2, t} d t\right] .
\end{aligned}
$$

At this stage, combining all the terms together, the second-order expansion for $g(1)$ (equation (C.8)) simply becomes

$$
g(1)=P_{B S}\left(x_{0}, \int_{0}^{T} v_{0, t}^{2} d t\right)+\mathbb{E}\left[\frac{\partial \tilde{P}_{B S}}{\partial y} \int_{0}^{T}\left(2 v_{0, t} V_{1, t}+V_{1, t}^{2}+2 v_{0, t} V_{2, t}\right) d t\right]+2 \mathbb{E}\left[\frac{\partial^{2} \tilde{P}_{B S}}{\partial y^{2}}\left\{\int_{0}^{T} v_{0, t} V_{1, t} d t\right\}^{2}\right]+\mathcal{E}
$$


Equation (C.14) is much simpler than equation (C.8), but still contains the stochastic processes $V_{1, t}$ and $V_{2, t}$. The last part of the proof is to simplify (C.14) further to get an expansion with explicit, deterministic coefficients.

Recall the short-hand notation for deterministic integrals from equations (3.8) and (3.9):

$$
\begin{gathered}
\omega_{t, T}^{(\kappa, l)}=\int_{t}^{T} e^{\int_{0}^{u} \kappa_{z} d z} l_{u} d u \quad \forall t \in[0, T] \\
\omega_{t, T}^{\left(\kappa_{n}, l_{n}\right), \ldots,\left(\kappa_{1}, l_{1}\right)}=\omega_{t, T}^{\left(\kappa_{n}, l_{n} \omega_{., T}^{\left.\left(\kappa_{n-1}, l_{n-1}\right), \ldots, \kappa_{1}, l_{1}\right)}\right)} \quad \forall t \in[0, T] .
\end{gathered}
$$

We will use extensively the following Lemma:

Lemma C.2. (Lemma 5.4 in Benhamou et al. [2010]) For any deterministic integrable function $f$ and any continuous semimartingale $Z$ such that $Z_{0}=0$,

$$
\int_{0}^{T} f(t) Z_{t} d t=\int_{0}^{T} \omega_{t, T}^{(0, f)} d Z_{t}
$$

Proof. Apply Itō's lemma to the product $\omega_{t, T}^{(0, f)} Z_{t}$.

The computation of each type of expectation in equation (C.14) is summarized in the following Lemma.

Lemma C.3. The following equalities hold

$$
\begin{aligned}
& \mathbb{E} {\left[l\left(\int_{0}^{T} \rho_{t} v_{0, t} d B_{t}\right) \int_{0}^{T} \beta_{t} V_{1, t} d t\right]=\omega_{0, T}^{\left(\kappa, \rho \lambda v_{0, .}^{2}\right),(-\kappa, \beta)} \mathbb{E}\left[l^{(1)}\left(\int_{0}^{T} \rho_{t} v_{0, t} d B_{t}\right)\right] } \\
& \mathbb{E}\left[l\left(\int_{0}^{T} \rho_{t} v_{0, t} d B_{t}\right) \int_{0}^{T} \beta_{t} V_{2, t} d t\right]=\omega_{0, T}^{\left(\kappa, \rho \lambda v_{0, .}^{2}\right),\left(0,2 \rho \lambda v_{0, .}\right),(-\kappa, \beta)} \mathbb{E}\left[l^{(2)}\left(\int_{0}^{T} \rho_{t} v_{0, t} d B_{t}\right)\right] \\
& \mathbb{E}\left[l\left(\int_{0}^{T} \rho_{t} v_{0, t} d B_{t}\right) \int_{0}^{T} \beta_{t} V_{1, t}^{2} d t\right]=\omega_{0, T}^{\left(2 \kappa, \lambda^{2} v_{0, .}^{2},(-2 \kappa, \beta)\right.} \mathbb{E}\left[l\left(\int_{0}^{T} \rho_{t} v_{0, t} d B_{t}\right)\right] \\
&+2 \omega_{0, T}^{\left(\kappa, \rho \lambda v_{0, .}^{2}\right),\left(\kappa, \rho \lambda v_{0, .}^{2}\right),(-2 \kappa, \beta)} \mathbb{E}\left[l^{(2)}\left(\int_{0}^{T} \rho_{t} v_{0, t} d B_{t}\right)\right] \\
& \mathbb{E}\left[l\left(\int_{0}^{T} \rho_{t} v_{0, t} d B_{t}\right)\left\{\int_{0}^{T} \beta_{t} V_{1, t} d t\right\}^{2}\right]=2 \omega_{0, T}^{\left(2 \kappa, \lambda^{2} v_{0, .}^{2},(-\kappa, \beta),(-\kappa, \beta)\right.} \mathbb{E}\left[l\left(\int_{0}^{T} \rho_{t} v_{0, t} d B_{t}\right)\right] \\
&+\left\{\omega_{0, T}^{\left(\kappa, \rho \lambda v_{0, .}^{2}\right),(-\kappa, \beta)}\right\}^{2} \mathbb{E}\left[l^{(2)}\left(\int_{0}^{T} \rho_{t} v_{0, t} d B_{t}\right)\right]
\end{aligned}
$$

where $\beta$, a deterministic function, and $l$, a twice-differentiable function, are such that these expectations exist.

Proof. Using Lemma C.2 and equation (C.13),

$$
\begin{aligned}
\mathbb{E}\left[l\left(\int_{0}^{T} \rho_{t} v_{0, t} d B_{t}\right) \int_{0}^{T} \beta_{t} V_{1, t} d t\right] & =\mathbb{E}\left[l\left(\int_{0}^{T} \rho_{t} v_{0, t} d B_{t}\right) \int_{0}^{T} \beta_{t} e^{-\int_{0}^{t} \kappa_{z} d z} \int_{0}^{t} e^{\int_{0}^{s} \kappa_{z} d z} \lambda_{s} v_{0, s} d B_{s} d t\right] \\
& =\mathbb{E}\left[l\left(\int_{0}^{T} \rho_{t} v_{0, t} d B_{t}\right) \int_{0}^{T} \omega_{t, T}^{(-\kappa, \beta)} e^{\int_{0}^{t} \kappa_{z} d z} \lambda_{t} v_{0, t} d B_{t}\right] \\
& =\omega_{0, T}^{\left(\kappa, \rho \lambda v_{0, .}^{2}\right),(-\kappa, \beta)} \mathbb{E}\left[l^{(1)}\left(\int_{0}^{T} \rho_{t} v_{0, t} d B_{t}\right)\right]
\end{aligned}
$$




$$
\begin{aligned}
& \mathbb{E}\left[l\left(\int_{0}^{T} \rho_{t} v_{0, t} d B_{t}\right) \int_{0}^{T} \beta_{t} V_{2, t} d t\right]=\mathbb{E}\left[l\left(\int_{0}^{T} \rho_{t} v_{0, t} d B_{t}\right) \int_{0}^{T} \beta_{t} e^{-\int_{0}^{t} \kappa_{z} d z} \int_{0}^{t} e^{\int_{0}^{s} \kappa_{z} d z} 2 \lambda_{s} V_{1, s} d B_{s} d t\right] \\
& =\mathbb{E}\left[l\left(\int_{0}^{T} \rho_{t} v_{0, t} d B_{t}\right) \int_{0}^{T} \omega_{t, T}^{(-\kappa, \beta)} e^{\int_{0}^{t} \kappa_{z} d z} 2 \lambda_{t} V_{1, t} d B_{t}\right]=\mathbb{E}\left[l^{(1)}\left(\int_{0}^{T} \rho_{t} v_{0, t} d B_{t}\right) \int_{0}^{T} \omega_{t, T}^{(-\kappa, \beta)} e^{\int_{0}^{t} \kappa_{z} d z} 2 \rho_{t} \lambda_{t} v_{0, t} V_{1, t} d t\right] \\
& =\mathbb{E}\left[l^{(1)}\left(\int_{0}^{T} \rho_{t} v_{0, t} d B_{t}\right) \int_{0}^{T} \omega_{t, T}^{(-\kappa, \beta)} 2 \rho_{t} \lambda_{t} v_{0, t} \int_{0}^{t} e^{\int_{0}^{s} \kappa_{z} d z} \lambda_{s} v_{0, s} d B_{s} d t\right] \\
& =\mathbb{E}\left[l^{(1)}\left(\int_{0}^{T} \rho_{t} v_{0, t} d B_{t}\right) \int_{0}^{T} \omega_{t, T}^{\left(0,2 \rho \lambda v_{0, .}\right),(-\kappa, \beta)} e^{\int_{0}^{t} \kappa_{z} d z} \lambda_{t} v_{0, t} d B_{t}\right]=\omega_{0, T}^{\left(\kappa, \rho \lambda v_{0, .}^{2}\right),\left(0,2 \rho \lambda v_{0, .}\right),(-\kappa, \beta)} \mathbb{E}\left[l^{(2)}\left(\int_{0}^{T} \rho_{t} v_{0, t} d B_{t}\right)\right] .
\end{aligned}
$$

Using (C.12),

$$
\begin{aligned}
& \mathbb{E}\left[l\left(\int_{0}^{T} \rho_{t} v_{0, t} d B_{t}\right) \int_{0}^{T} \beta_{t} V_{1, t}^{2} d t\right]=\mathbb{E}\left[l\left(\int_{0}^{T} \rho_{t} v_{0, t} d B_{t}\right) \int_{0}^{T} \beta_{t} e^{-2 \int_{0}^{t} \kappa_{z} d z}\left\{\int_{0}^{t} e^{\int_{0}^{s} \kappa_{z} d z} \lambda_{s} v_{0, s} d B_{s}\right\}^{2} d t\right] \\
& =\mathbb{E}\left[l\left(\int_{0}^{T} \rho_{t} v_{0, t} d B_{t}\right) \int_{0}^{T} \beta_{t} e^{-2 \int_{0}^{t} \kappa_{z} d z} \int_{0}^{t} 2\left\{\int_{0}^{s} e^{\int_{0}^{u} \kappa_{z} d z} \lambda_{u} v_{0, u} d B_{u}\right\} e^{\int_{0}^{s} \kappa_{z} d z} \lambda_{s} v_{0, s} d B_{s} d t\right] \\
& +\mathbb{E}\left[l\left(\int_{0}^{T} \rho_{t} v_{0, t} d B_{t}\right) \int_{0}^{T} \beta_{t} e^{-2 \int_{0}^{t} \kappa_{z} d z}\left\{\int_{0}^{t} e^{2 \int_{0}^{s} \kappa_{z} d z} \lambda_{s}^{2} v_{0, s}^{2} d s\right\} d t\right] .
\end{aligned}
$$

First, using Lemma C.2,

$$
\mathbb{E}\left[l\left(\int_{0}^{T} \rho_{t} v_{0, t} d B_{t}\right) \int_{0}^{T} \beta_{t} e^{-2 \int_{0}^{t} \kappa_{z} d z}\left\{\int_{0}^{t} e^{2 \int_{0}^{s} \kappa_{z} d z} \lambda_{s}^{2} v_{0, s}^{2} d s\right\} d t\right]=\omega_{0, T}^{\left(2 \kappa, \lambda^{2} v_{0, .}^{2}\right),(-2 \kappa, \beta)} \mathbb{E}\left[l\left(\int_{0}^{T} \rho_{t} v_{0, t} d B_{t}\right)\right] .
$$

Then, using Lemma C.2, Lemma (C.1) and then equation (C.15),

$$
\begin{aligned}
& \mathbb{E}\left[l\left(\int_{0}^{T} \rho_{t} v_{0, t} d B_{t}\right) \int_{0}^{T} \beta_{t} e^{-2 \int_{0}^{t} \kappa_{z} d z} \int_{0}^{t} 2\left\{\int_{0}^{s} e^{\int_{0}^{u} \kappa_{z} d z} \lambda_{u} v_{0, u} d B_{u}\right\} e^{\int_{0}^{s} \kappa_{z} d z} \lambda_{s} v_{0, s} d B_{s} d t\right] \\
& =\mathbb{E}\left[l\left(\int_{0}^{T} \rho_{t} v_{0, t} d B_{t}\right) \int_{0}^{T} 2 \omega_{t, T}^{(-2 \kappa, \beta)} e^{\int_{0}^{t} \kappa_{z} d z} \lambda_{t} v_{0, t}\left\{\int_{0}^{t} e^{\int_{0}^{s} \kappa_{z} d z} \lambda_{s} v_{0, s} d B_{s}\right\} d B_{t}\right] \\
& =\mathbb{E}\left[l^{(1)}\left(\int_{0}^{T} \rho_{t} v_{0, t} d B_{t}\right) \int_{0}^{T} 2 \omega_{t, T}^{(-2 \kappa, \beta)} e^{2 \int_{0}^{t} \kappa_{z} d z} \rho_{t} \lambda_{t} v_{0, t}^{2} V_{1, t} d t\right] \\
& =2 \omega_{0, T}^{\left(\kappa, \rho \lambda v_{0, .}^{2},\left(\kappa, \rho \lambda v_{0, .}^{2}\right),(-2 \kappa, \beta)\right.} \mathbb{E}\left[l^{(2)}\left(\int_{0}^{T} \rho_{t} v_{0, t} d B_{t}\right)\right],
\end{aligned}
$$

yielding (C.17). Finally, using (C.12) and Lemma C.2,

$$
\begin{aligned}
\mathbb{E}\left[l\left(\int_{0}^{T} \rho_{t} v_{0, t} d B_{t}\right)\left\{\int_{0}^{T} \beta_{t} V_{1, t} d t\right\}^{2}\right] & =\mathbb{E}\left[l\left(\int_{0}^{T} \rho_{t} v_{0, t} d B_{t}\right) 2 \int_{0}^{T}\left\{\int_{0}^{t} \beta_{s} V_{1, s} d s\right\} \beta_{t} V_{1, t} d t\right] \\
& =2 \mathbb{E}\left[l\left(\int_{0}^{T} \rho_{t} v_{0, t} d B_{t}\right) \int_{0}^{T} \omega_{t, T}^{(-\kappa, \beta)} e^{\int_{0}^{t} \kappa_{z} d z} \beta_{t} V_{1, t}^{2} d t\right] \\
& +2 \mathbb{E}\left[l\left(\int_{0}^{T} \rho_{t} v_{0, t} d B_{t}\right) \int_{0}^{T} \omega_{t, T}^{(-\kappa, \beta)}\left\{\int_{0}^{t} \beta_{s} V_{1, s} d s\right\} e^{\int_{0}^{t} \kappa_{z} d z} \lambda_{t} v_{0, t} d B_{t}\right]
\end{aligned}
$$


Using equation (C.17),

$$
\begin{aligned}
2 \mathbb{E}\left[l\left(\int_{0}^{T} \rho_{t} v_{0, t} d B_{t}\right) \int_{0}^{T} \omega_{t, T}^{(-\kappa, \beta)} e^{\int_{0}^{t} \kappa_{z} d z} \beta_{t} V_{1, t}^{2} d t\right] & =2 \omega_{0, T}^{\left(2 \kappa, \lambda^{2} v_{0, .}^{2}\right),(-\kappa, \beta),(-\kappa, \beta)} \mathbb{E}\left[l\left(\int_{0}^{T} \rho_{t} v_{0, t} d B_{t}\right)\right] \\
& +4 \omega_{0, T}^{\left(\kappa, \rho \lambda v_{0, .}^{2}\right),\left(\kappa, \rho \lambda v_{0, .}^{2}\right),(-\kappa, \beta),(-\kappa, \beta)} \mathbb{E}\left[l^{(2)}\left(\int_{0}^{T} \rho_{t} v_{0, t} d B_{t}\right)\right] .
\end{aligned}
$$

Then, using Lemma C.1, Lemma C.2, and equation (C.15),

$$
\begin{aligned}
& 2 \mathbb{E}\left[l\left(\int_{0}^{T} \rho_{t} v_{0, t} d B_{t}\right) \int_{0}^{T} \omega_{t, T}^{(-\kappa, \beta)}\left\{\int_{0}^{t} \beta_{s} V_{1, s} d s\right\} e^{\int_{0}^{t} \kappa_{z} d z} \lambda_{t} v_{0, t} d B_{t}\right] \\
& =2 \mathbb{E}\left[l^{(1)}\left(\int_{0}^{T} \rho_{t} v_{0, t} d B_{t}\right) \int_{0}^{T} \omega_{t, T}^{(-\kappa, \beta)} e^{\int_{0}^{t} \kappa_{z} d z} \rho_{t} \lambda_{t} v_{0, t}^{2}\left\{\int_{0}^{t} \beta_{s} V_{1, s} d s\right\} d t\right] \\
& =2 \mathbb{E}\left[l^{(1)}\left(\int_{0}^{T} \rho_{t} v_{0, t} d B_{t}\right) \int_{0}^{T} \omega_{t, T}^{\left(\kappa, \rho \lambda v_{0, .}^{2}\right),(-\kappa, \beta)} \beta_{t} V_{1, t} d t\right] \\
& =2 \omega_{0, T}^{\left(\kappa, \rho \lambda v_{0, .}^{2}\right),(-\kappa, \beta),\left(\kappa, \rho \lambda v_{0, .}^{2}\right),(-\kappa, \beta)} \mathbb{E}\left[l^{(2)}\left(\int_{0}^{T} \rho_{t} v_{0, t} d B_{t}\right)\right],
\end{aligned}
$$

and using that $4 \omega_{0, T}^{\left(\kappa, \rho \lambda v_{0, .}^{2}\right),\left(\kappa, \rho \lambda v_{0, .}^{2}\right),(-\kappa, \beta),(-\kappa, \beta)}+2 \omega_{0, T}^{\left(\kappa, \rho \lambda v_{0, .}^{2},\right),(-\kappa, \beta),\left(\kappa, \rho \lambda v_{0, .}^{2}\right),(-\kappa, \beta)}=\left\{\omega_{0, T}^{\left(\kappa, \rho \lambda v_{0, .}^{2}\right),(-\kappa, \beta)}\right\}^{2}$

(Benhamou et al. [2010] p. 34) yields equation (C.18).

To conclude the proof, apply Lemma C.3 and equation (C.10) to each term of equation (C.14), yielding

$$
\begin{aligned}
g(1) & =P_{B S}\left(x_{0}, \int_{0}^{T} v_{0, t}^{2} d t\right)+\mathbb{E}\left[\frac{\partial \tilde{P}_{B S}}{\partial y} \int_{0}^{T}\left(2 v_{0, t} V_{1, t}+V_{1, t}^{2}+2 v_{0, t} V_{2, t}\right) d t\right]+2 \mathbb{E}\left[\frac{\left.\partial^{2} \tilde{P}_{B S}\left\{\int_{0}^{T} v_{0, t} V_{1, t} d t\right\}^{2}\right]}{}=P_{B S}\left(x_{0}, \int_{0}^{T} v_{0, t}^{2} d t\right)\right. \\
& +\omega_{0, T}^{\left(\kappa, \rho \lambda v_{0, .}^{2}\right),\left(-\kappa, 2 v_{0, .}\right)} \frac{\partial^{2} P_{B S}}{\partial x \partial y}\left(x_{0}, \int_{0}^{T} v_{0, t}^{2} d t\right) \\
& +\omega_{0, T}^{\left(2 \kappa, \lambda^{2} v_{0, .}^{2},\right),(-2 \kappa, 1)} \frac{\partial P_{B S}}{\partial y}\left(x_{0}, \int_{0}^{T} v_{0, t}^{2} d t\right) \\
& +2 \omega_{0, T}^{\left(\kappa, \rho \lambda v_{0, .}^{2}\right),\left(\kappa, \rho \lambda v_{0, .}^{2}\right),(-2 \kappa, 1)} \frac{\partial^{3} P_{B S}}{\partial x^{2} \partial y}\left(x_{0}, \int_{0}^{T} v_{0, t}^{2} d t\right) \\
& +\omega_{0, T}^{\left(\kappa, \rho \lambda v_{0, .}^{2}\right),\left(0,2 \rho \lambda v_{0, .}\right),\left(-\kappa, 2 v_{0, .}\right)} \frac{\partial^{3} P_{B S}}{\partial x^{2} \partial y}\left(x_{0}, \int_{0}^{T} v_{0, t}^{2} d t\right) \\
& +4 \omega_{0, T}^{\left(2 \kappa, \lambda^{2} v_{0, .}^{2}\right),\left(-\kappa, v_{0, .}\right),\left(-\kappa, v_{0, .}\right)} \frac{\partial^{2} P_{B S}}{\partial y^{2}}\left(x_{0}, \int_{0}^{T} v_{0, t}^{2} d t\right) \\
& +2\left\{\omega_{0, T}^{\left(\kappa, \rho \lambda v_{0, .}^{2}\right),\left(-\kappa, v_{0, .}\right)}\right\}^{2} \frac{\partial^{4} P_{B S}}{\partial x^{2} \partial y^{2}}\left(x_{0}, \int_{0}^{T} v_{0, t}^{2} d t\right) \\
& +\mathcal{E},
\end{aligned}
$$

which corresponds to the announced Theorem 3.1. 